\title{
Morphology, Aggregation Properties, Cytocompatibility, and Anti-Inflammatory Potential of Citrate-Stabilized AuNPs Prepared by Modular Ultrasonic Spray Pyrolysis
}

\author{
Rebeka Rudolf, ${ }^{1,2}$ Peter Majerič, ${ }^{1}$ Sergej Tomić, ${ }^{3}$ Mohammed Shariq, ${ }^{1,4}$ Urban Ferčec, ${ }^{5}$ \\ Bojan Budič, ${ }^{6}$ Bernd Friedrich, ${ }^{7}$ Dragana Vučević, ${ }^{8}$ and Miodrag Čolićc ${ }^{3,8}$ \\ ${ }^{1}$ Institute of Materials Technology, University of Maribor, SI-2000 Maribor, Slovenia \\ ${ }^{2}$ Zlatarna Celje d.d., SI-3000 Celje, Slovenia \\ ${ }^{3}$ Institute for the Application of Nuclear Energy, University of Belgrade, 11000 Belgrade, Serbia \\ ${ }^{4}$ Indian Institute of Technology (ISM), Dhanbad, Jharkhand 826 004, India \\ ${ }^{5}$ Faculty of Chemistry and Chemical Technology, University of Ljubljana, SI-1000 Ljubljana, Slovenia \\ ${ }^{6}$ National Institute of Chemistry, SI-1000 Ljubljana, Slovenia \\ ${ }^{7}$ IME Institute, RWTH Aachen University, Aachen, Germany \\ ${ }^{8}$ Institute for Medical Research, Medical Faculty of the Military Medical Academy, University of Defense, 11000 Belgrade, Serbia \\ Correspondence should be addressed to Rebeka Rudolf; rebeka.rudolf@um.si
}

Received 27 July 2016; Revised 3 November 2016; Accepted 6 November 2016; Published 2 January 2017

Academic Editor: Ilaria Fratoddi

Copyright (C) 2017 Rebeka Rudolf et al. This is an open access article distributed under the Creative Commons Attribution License, which permits unrestricted use, distribution, and reproduction in any medium, provided the original work is properly cited.

\begin{abstract}
Ultrasonic Spray Pyrolysis (USP) possesses a great potential for production of higher quantities of gold nanoparticles (AuNPs), thus overcoming the problem of batch-to-batch variations in their properties. Recently, we demonstrated that USP with an additional evaporation chamber (modular USP) led to a better size control of AuNPs. However, their morphology, stability, toxicity, and immunomodulatory properties have not been investigated completely. Here, two types of spherical AuNPs were produced by using different USP parameters, followed by their stabilization in Na-citrate solution. No significant changes in their size, agglomeration, and $z$-potential occurred 3 months after their initial production in citrate solution. However, the conditioning of AuNPs in serumcontaining cell culture media for $24 \mathrm{~h}$ induced an increase in the AuNPs' hydrodynamic size and a red shift in their Surface Plasmon Resonance, pointing to their instability in biological media. Cytocompatibility tests showed that the produced AuNPs were internalized by L929 cells and primary human monocytes and were not cytotoxic at the concentrations lower than $200 \mu \mathrm{g} / \mathrm{mL}$, but they exhibited antiproliferative and anti-inflammatory effects, respectively. AuNPs reduced the percentage of CD14 ${ }^{+} \mathrm{CD} 16^{+}$but not $\mathrm{CD} 14{ }^{\text {low }} \mathrm{CD} 16^{+}$monocytes in vitro and reduced the expression of CD86, HLA-DR, TNF- $\alpha$, and IL-12/IL-23 by these cells. These results indicate that the anti-inflammatory effects of citrate-capped AuNPs produced by modular USP could be beneficial for their application in the treatment of inflammatory conditions.
\end{abstract}

\section{Introduction}

Gold nanoparticles (AuNPs) possess excellent Surface Plasmon (SP) properties, enabling tunable size-dependent optical properties of AuNPs. When the SP of AuNPs, that is, the oscillation of conduction electrons on the surface of the nanoparticles, is in resonance with the incident light, a strong nonfading emission can be obtained [1]. Shi et al. [2] and Jain et al. [3] reported that the magnitude of light scattering by
$80 \mathrm{~nm}$ AuNPs is five orders higher than the light emission from strong fluorescing dyes. Furthermore, AuNPs are much more efficient as the photon thermal energy converters than the typical organic molecules. AuNPs can also be functionalized easily with different biomolecules, all of which open the path for the development of efficient diagnostic tools and drug carriers [3-5]. Besides their great physical and chemical properties, AuNPs possess good biocompatibility, so, expectedly, the main markets for AuNPs are in biomedicine, tissue 


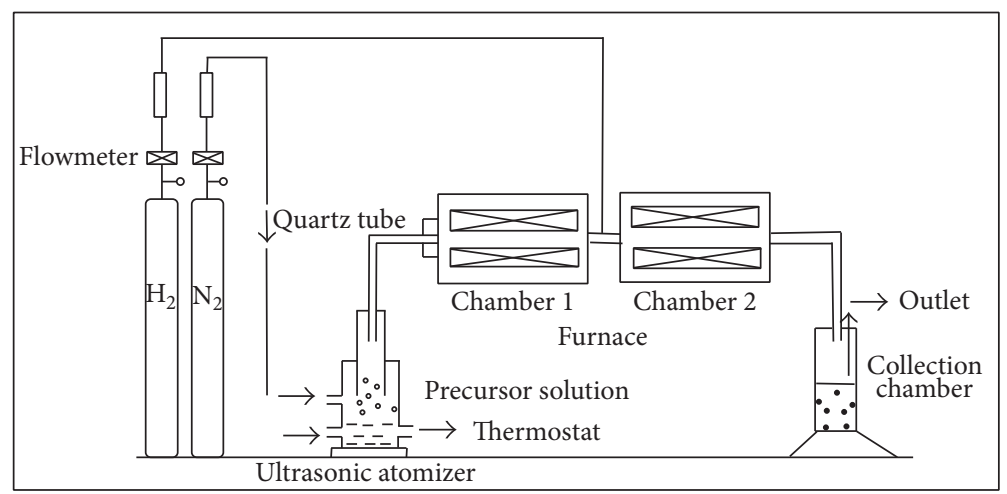

FIGURE 1: Schematic diagram of the modular USP device. The modular USP consisted of an ultrasonic atomizer for the generation of aerosol droplets, heating furnaces of chamber 1 and chamber 2 for the evaporation of droplets and particle drying, a collection chamber for collecting the nanoparticles, and quartz tubes for transportation of droplets and particles.

or tumor imaging, drug delivery, photothermal therapy, and immunochromatographic identification of pathogens in clinical specimens [6].

Different production methods for AuNPs have been developed such as bottom-up synthesis by sol-gel, chemical vapor deposition, flame spray synthesis, and atomic or molecular condensation [7-10]. Top-down methods include laser ablation, nanolithography, and high-energy milling [11, 12]. However, these methods are suitable for production of small quantities of AuNPs, leading to major variations in shapes and sizes between different batches, which hampers the implementation of clinical trials with AuNPs and their application in diagnostics and therapy. A promising bottomup method, called Ultrasonic Spray Pyrolysis (USP), has a good potential for removing these technological issues [9, 13]. In USP, the ultrasound is used for dispersion of the precursor solution into droplets, followed by their chemical decomposition at elevated temperatures $[14,15]$. The advantage of the USP method is the simplicity of setting up individual parameters and process segments, as well as the ability of continuous AuNPs synthesis. Additionally, we showed previously that USP could be applied for the generation of pure AuNPs, even from gold scrap [16, 17], which reduces the cost of AuNPs greatly. We also found that the synthesis of AuNPs with USP enables the production of different sizes and shapes (spheres, discs, and triangles) of pure AuNPs from chloroauric precursor [18, 19]. However, due to the nonlinear dynamics of the USP process, the control of the shape and size of AuNPs was not achieved completely. Therefore, we have redesigned the USP apparatus recently with an additional heating chamber and obtained much better control over the synthesis of AuNPs, compared to the conventional USP [20]. Furthermore, AuNPs obtained by this modular USP showed spherical shape and lower size range, and they were not toxic for L929 cells at a concentration of $100 \mu \mathrm{g} / \mathrm{mL}$ during $48 \mathrm{~h}$ exposure [21]. However, we also observed that AuNPs collected in deionized water by modular USP agglomerate/aggregate after prolonged storage. Therefore, here we introduced additionally the stabilization of AuNPs at the collection stage by using Na-citrate solution.
However, it is not known whether the citrate stabilization of such AuNPs could affect their cytocompatibility. Although citrate is considered as a good and nontoxic AuNP stabilizer $[22,23]$, it has been reported that the citrate-stabilized AuNPs applied in high concentrations could affect the actin cytoskeleton and the proliferation of fibroblasts [24] and HEPG2 cells [25]. Additionally, we showed previously that nontoxic AuNPs produced by USP [16], similar to those obtained commercially [26], can induce immunomodulatory effects by modulating the functions of Antigen Presenting Cells (APCs). Monocytes represent a major population of phagocytic APCs in blood, and they give rise to the tissue macrophages and inflammatory DCs [27], both of which are responsible for immune and inflammatory responses to nanoparticles in tissues [28]. However, the data on the effects of citrate-capped AuNPs on human monocytes are quite scarce. Therefore, besides the cytotoxicity evaluation, we investigated the response of primary human monocytes to citrate-capped AuNPs produced by modular USP.

\section{Experimental Details}

2.1. Synthesis of Gold Nanoparticles. The synthesis of AuNPs was carried out on the modular USP device at the IME Institute of Process Metallurgy and Metal Recycling, RWTH Aachen University, Germany (Figure 1). An aqueous solution of Hydrogen Tetrachloroaurate (either $2.5 \mathrm{~g} / \mathrm{L}$ or $0.5 \mathrm{~g} / \mathrm{L}$, Sigma-Aldrich) in deionized water (Millipore) was used as the precursor. The prepared precursor solutions were fed into the Ultrasonic Aerosol Generator (Gapusol, RBI, France, piezoelectric transducer membrane frequency: $2.5 \mathrm{MHz}$ ) to form aerosol droplets with diameters ranging from 1 to $15 \mu \mathrm{m}$ [29]. A nitrogen gas flow range of 1.0 to $4.5 \mathrm{~L} / \mathrm{min}$ was used as the carrier gas to transport these formed aerosol droplets to the two heating zones through a quartz glass tube of $2 \mathrm{~cm}$ diameter. A hydrogen gas flow, set to 1.0 to $2.0 \mathrm{~L} / \mathrm{min}$, was added as a reducing agent for the gold chloride solution to pure gold metal nanoparticles. The first heating zone was set at a dispersed temperature range from 50 to $100^{\circ} \mathrm{C}$ for droplets' evaporation and particle drying and also allowed 
TABLE 1: Parameters for the synthesis of AuNPs.

\begin{tabular}{lccccccc}
\hline Sample name $[\mathrm{Au}](\mathrm{g} / \mathrm{L})$ & $T_{1}\left({ }^{\circ} \mathrm{C}\right)$ & $T_{2}\left({ }^{\circ} \mathrm{C}\right)$ & $N_{2}(\mathrm{~L} / \mathrm{min})$ & $H_{2}(\mathrm{~L} / \mathrm{min})$ & Duration of the experiment (hours) & Solution consumed $(\mathrm{mL})$ \\
\hline AuC18 & 2.5 & 90 & 350 & 1.5 & 1 & 3 & 60 \\
AuC20 & 0.5 & 90 & 350 & 4.5 & 2.0 & 3 & 60 \\
\hline
\end{tabular}

an optimal material diffusion inside the droplet ([AuCl4]and $\mathrm{H}+$ ). The second reactor furnace was set at a temperature range from 260 to $500^{\circ} \mathrm{C}$ and was responsible for the chemical reactions required for obtaining pure AuNPs. The ambient temperature was $21^{\circ} \mathrm{C}$. The two sets of parameters (Table 1) enabled generation of two types of AuNPs (AuC18 and AuC20: "Au" is for gold nanoparticles, "C" is the series separator, and "18" and " 20 " are the experiments in the series). The parameters presented in this article have been chosen based on the results of a series of experiments, while these have yielded the best results for our target nanoparticles. The nanoparticles were collected in $0.1 \%$ solution of Sodium Citrate (Alfa Aesar) in Millipore water ( $\mathrm{pH} 3.5 \pm 1$ ). The lack of $\mathrm{pH}$ change after the collection of AuNPs in citrate solution indicated that $\mathrm{Au}$ was reduced completely by the hydrogen gas flow rather than by the citrate in the collection bottle. All synthesized AuNPs were stored in Miron glass at $8^{\circ} \mathrm{C}$ away from light for at least 3 months.

\subsection{Characterization Technique}

2.2.1. TEM. Conventional Transmission Electron Microscopy (CTEM; JEOL 2100), High-Resolution Transmission Electron Microscopy (HRTEM; JEOL 2100), Electron Diffraction (ED/TEM; JEOL 2100), and Energy Dispersive Spectroscopy (EDS/TEM; JED-2300) investigations were conducted on the prepared AuNPs to study the morphology, crystal lattice, and chemical composition of the obtained AuNPs. A drop of colloidal suspension of AuNPs was pipetted onto a formvar film coated with a layer of carbon or a lacey formvar film enforced by a heavy coating of carbon TEM copper grid of 200 meshes and dried at room temperature. The grid was then observed directly in a TEM once the medium evaporated. The size and circularity measurements of AuNPs were done on TEM images by using a minimum of 200 discrete AuNPs from each of widely separated regions of the two samples, according to Standard ISO 13322-1:2004. ImageJ software tool was used for the data analysis algorithm.

\subsubsection{Inductively Coupled Plasma Optical Emission Spectrom-} etry. Inductively Coupled Plasma Optical Emission Spectrometry (ICP-OES) was used for the $\mathrm{Au}^{3+}$ quantification (i.e., the concentration of Au in AuNPs). Prior to the analysis, samples were diluted 10 -fold with deionized Milli-Q water (purity $18 \mathrm{M} \Omega \mathrm{cm}$ ) and acidified with aqua regia $(5 \% \mathrm{v} / \mathrm{v})$. For calibration, single element standard solutions (Merck, Darmstadt, Germany) were used. Analysis was carried out using an ICP-MS spectrometer (Agilent, 7500ce, equipped with collision cell) under the operating conditions: RF power, $1.5 \mathrm{~kW}$, sample depth, $8 \mathrm{~mm}$, nebulizer, Meinhard, plasma gas flow (L/min), 15 , nebulizer gas flow (L/min), 0.85 , makeup gas flow $(\mathrm{L} / \mathrm{min}), 0.28$, and reaction gas flow $(\mathrm{mL} / \mathrm{min}), 4.0$.
2.2.3. DLS and UV-Vis Measurements. The AuNPs' size and zeta-potential were obtained using a Malvern (Multipurpose Titrator) Zetasizer Nano ZS. During the automatic measurements (10-30 runs), the initial parameters for absorption (0.010), refractive index (1.59), dispersant properties (water), temperature $\left(25^{\circ} \mathrm{C}\right)$, equilibration time $(25 \mathrm{~s})$, measurement angle ( $173^{\circ}$ backscatter), and cell type (Dip Cell) were set for zeta-potential measurements. In some experiments, AuNPs were first concentrated $200 \mathrm{x}$ by centrifugation $(2000 \times \mathrm{g}, 20$ minutes), followed by their dispersion in an ultrasonic bath for 15 minutes, prior to the conditioning experiments and cytocompatibility testing.

In the conditioning experiments, dispersed AuNPs $(50 \mu \mathrm{g} / \mathrm{mL})$ were incubated in a complete RPMI medium (basic RPMI 1640 (Sigma-Aldrich), supplemented with 10\% fetal bovine serum (Gibco), 2-mercaptoethanol (Sigma), penicillin, streptomycin, and gentamicin (1\% each, ICN Galeni$\mathrm{ka})$ ) for $24 \mathrm{~h}$ and then analyzed by Zeta Nano ZS, as described.

The UV-vis spectra of AuNPs dispersed in $0.03 \% \mathrm{Na}$ citrate solution and in complete RPMI medium (1:2 AuNP in $0.1 \% \mathrm{Na}$-citrate: medium vol. ratio) for $2 \mathrm{~h}$ or $24 \mathrm{~h}$ were analyzed using Ultrospec 2000 (Pharmacia Biotech) within $\lambda$ range of $200-900 \mathrm{~nm}$. The blank samples, $0.03 \%$ citrate solution and corresponding concentrations of citrate in complete RPMI medium, were used for background subtraction. The SPR curves detected between 400 and $700 \mathrm{~nm}$ were averaged from 10 measurements and normalized to peak value 1 in each sample to enable comparison between samples.

2.3. L929 Cells. The following cells were used in the cytocompatibility assays: L929 cells and primary human monocytes. L929, a mouse fibroblast cell line, was obtained from ATCC (Washington DC, USA). Prior to the experiments, L929 cells were thawed from liquid nitrogen and cultivated in complete RPMI medium at $37^{\circ} \mathrm{C}, 5 \% \mathrm{CO}_{2}$, until they reached $90 \%$ confluence. After that, the cells were trypsinized with $0.25 \%$ trypsin solution (Sigma) in 0.02\% NaEDTA/RPMI. After two passages, L929 cells were seeded in 96 -well plate $\left(1 \times 10^{4} /\right.$ well $)$ for $24 \mathrm{~h}$, washed with complete RPMI medium, and then treated with AuC18 or AuC20 NPs (12.5-200 $\mu \mathrm{g} / \mathrm{mL})$.

The viability of L929 cells was determined after $24 \mathrm{~h}$ by harvesting all cells and staining them with $1 \%$ Trypan blue. The labeled cells, identified by light microscopy, were considered as dead, predominantly necrotic cells. The percentages of dead cells were determined on the basis of at least 500 total cells from one well. The percentage of viable cells was calculated as $100 \%$ of total cells - $\%$ of dead cells.

Additionally, the percentage of apoptotic/necrotic L929 cells after the cultures was determined by Annexin-VFITC/Propidium Iodide (PI) labelling kit (R\&D Systems) and analyzing them on a flow cytometer (Partec Cube 6). 
An MTT assay was performed to determine the metabolic activity of L929 cells cultivated with AuNPs. L929 cells (2 $\times 10^{4} /$ well of 96 -well plate) were cultivated overnight to reach subconfluence after which they were washed with complete RPMI medium twice and then treated with AuC18 or AuC20 NPs $(12.5-200 \mu \mathrm{g} / \mathrm{mL}$ in medium) or medium alone (nontreated control), in 6-plicates for the next $24 \mathrm{~h}$. The corresponding cell-free cultures were prepared as blank controls. After the cultivation, all cultures were washed in phenol-red free RPMI medium twice to remove free AuNPs, and a tetrazolium dye MTT 3-(4,5-dimethylthiazol-2-yl)-2,5diphenyltetrazolium bromide $(1 \mathrm{mg} / \mathrm{mL}$ in phenol-red free RPMI) was added for the next $4 \mathrm{~h}$. The formazan crystals generated by NADH-oxidoreductase enzymes were dissolved by using $10 \%$ Sodium Dodecyl Sulphate (SDS) in $0.01 \mathrm{~N} \mathrm{HCl}$ overnight, and the absorbance was read at $570 \mathrm{~nm}$ (Behring ELISA Processor II, Heidelberg, Germany). The absorbance measured in cell-free blank controls was subtracted from the absorbance of corresponding experimental cultures. The absorbance (metabolic activity) detected in the treated cultures was expressed as the percentage of absorbance in the nontreated control cultures (100\%).

The proliferation of L929 cells (seeded initially at $0.5 \times$ $10^{4} /$ well) which were cultivated with AuNPs (12.5$200 \mu \mathrm{g} / \mathrm{mL}$ ) was determined after 3 days of cultivation. During the last 18 hours, the cells were pulsed with $1 \mu \mathrm{Ci} /$ well $[3 \mathrm{H}]$ thymidine $(6.7 \mathrm{Ci} / \mathrm{mmol}$, Amersham, Bucks, UK) to estimate the level of DNA synthesis in the log-phase of cellular proliferation. The cells were harvested onto glass fiber filters and the incorporation of the radionuclide into DNA was measured by $\beta$-scintillation counting (LKB-1219 Rackbeta, Finland).

2.4. Primary Human Monocytes. Primary human monocytes were isolated from Peripheral Blood Mononuclear Cells (PBMCs) from healthy volunteers who provided consent forms. All studies on human blood cells were approved by the Ethical Committee of the Military Medical Academy. PBMCs $\left(10^{6}\right.$ cells $\left./ \mathrm{cm}^{2}\right)$ were allowed to adhere for 1.5 hours, after which nonadherent cells were washed out thoroughly with prewarmed RPMI medium. After that, AuC18 or AuC20 NPs were added to the cell cultures. Additionally, some cultures were treated simultaneously with LPS $(500 \mathrm{ng} / \mathrm{mL})$ to induce the activation of monocytes. After $24 \mathrm{~h}$ of cultivation, the internalization of AuNPs by monocytes was studied, as well as the apoptosis/necrosis of monocytes and their phenotypic properties.

For the internalization studies, cultivated monocytes were harvested, washed in PBS solution, and added to a microscopic slide prior to their analysis by phase contrast microscopy. Alternatively, the cells were analyzed by flow cytometry (Partec Cube 6) to assess the granularity of the cells by monitoring the Side Scatter (SS) parameter.

Apoptosis/necrosis of the cultivated monocytes was determined by Annexin-V-FITC/Propidium Iodide (PI) staining kit (R\&D Systems), according to the manufacturer's protocol.

The phenotypic properties of LPS-stimulated and nonstimulated monocytes cultivated with AuNPs were assessed by flow cytometry using the following antibodies (Abs)/reagents: IgG1 negative control-phycoerythrin (PE), anti-CD14-FITC, anti-CD86-FITC, IgG1 negative control-FITC, anti-HLADR biotin, IgGla negative control-PE-Cy5 (eBioscience, Frankfurt, Germany), anti-CD16-PE (BioLegend, London, UK), anti-TNF- $\alpha$-FITC, anti-p40-PE, and streptavidin-PECy5 (R\&D). For intracellular labeling (detection of TNF- $\alpha$ and p40) a flow cytometry fixation and permeabilization kit (R\&D) was used, according to the manufacturer's protocol. Isotype control antibodies were used to determine nonspecific staining, and the cells labeled with single antibodies were used to compensate overlapping signals in each experiment.

2.5. Statistics. Data are presented as a representative experiment or as a mean \pm Standard Deviation (SD) of at least 3 independent experiments. The differences between control experimental samples were analyzed using the Friedman test with Dunn's posttest, and the values with $p<0.05$ or less were considered to be statistically significant.

\section{Results and Discussion}

3.1. Properties of USP-Generated Citrate-Stabilized AuNPs. USP has a good potential for removing technological issues such as small production rates with major variations in shape and sizes of the nanoparticles, but additional improvements of the process are required to limit the size variability of AuNPs and to improve their stability. In this paper, we applied an innovation by separating the heating chambers of the UPS into the evaporation/drying chamber for drying of the droplets generated by the ultrasound $(2.5 \mathrm{MHz})$ and the thermal decomposition chamber (Figure 1), leading to better control of AuNP size [21]. Considering that the dynamics of droplets formation depend strongly on the precursor concentrations and the gas flow rate $[18,19]$, we applied two sets of parameters (Table 1) to generate two types of AuNPs (AuC18 and $\mathrm{AuC20}$ ), followed by their stabilization in Na-citrate solution. ICP-OES measurements of $\mathrm{Au}^{3+}$ in the collection citrate solution suggested that the concentrations of $\mathrm{AuC18}$ and AuC20 NPs were $14 \mu \mathrm{g} / \mathrm{L}$ and $4.1 \mu \mathrm{g} / \mathrm{L}$, respectively.

To analyze the size and morphology of AuC18 and AuC20, first we conducted TEM analysis on freshly synthesized samples and on those stored in the citrate solution for 3 months (Figure 2). It was observed that AuNPs possess predominantly circular shape with no visible defects (cracks, pores, etc.). The circularity of $\mathrm{AuC1} 18$ and AuC20 samples was $0.86 \pm 0.03$ and $0.88 \pm 0.02$, respectively $(0$ signifies the irregular shape and 1 signifies the perfect circle), and the density of AuNPs was uniform within each sample. The average core size of AuC18 was $14.7 \pm 13.9 \mathrm{~nm}(N=200)$ with median (range) at $9.3(3.7-76.6) \mathrm{nm}$. The average core size of AuC20 was $36.8 \pm 10.4 \mathrm{~nm}(N=212)$ with median (range) at $35.1(15.2-88.2) \mathrm{nm}$ (Figure 2(a)). Similar results were obtained on the samples stored in citrate buffer for 3 months, suggesting that AuNPs had a stable size and morphology during the storage (Figure 2(b)). Based on the ICP-OES detected concentration of $\mathrm{Au}$ and the average size of AuC18 and AuC20 NPs observed by TEM, the theoretical number 

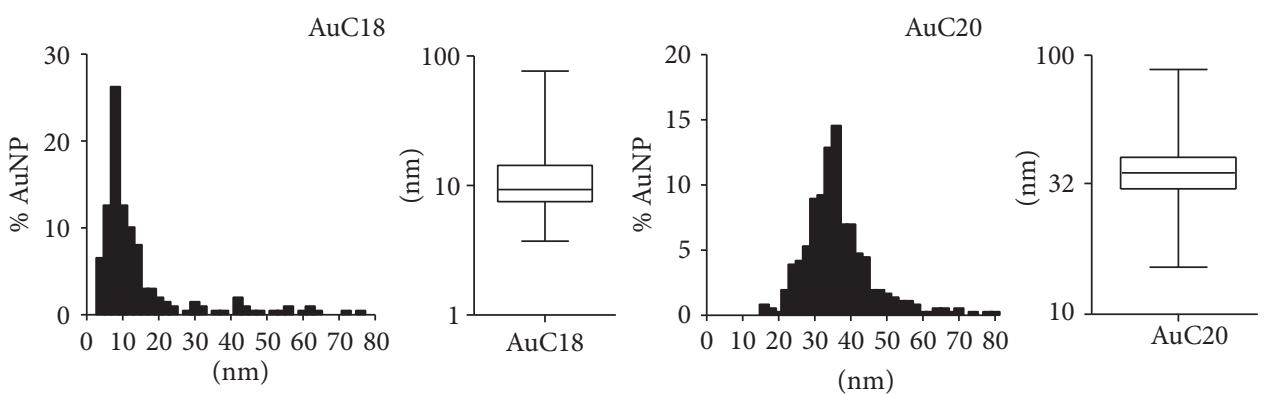

(a)
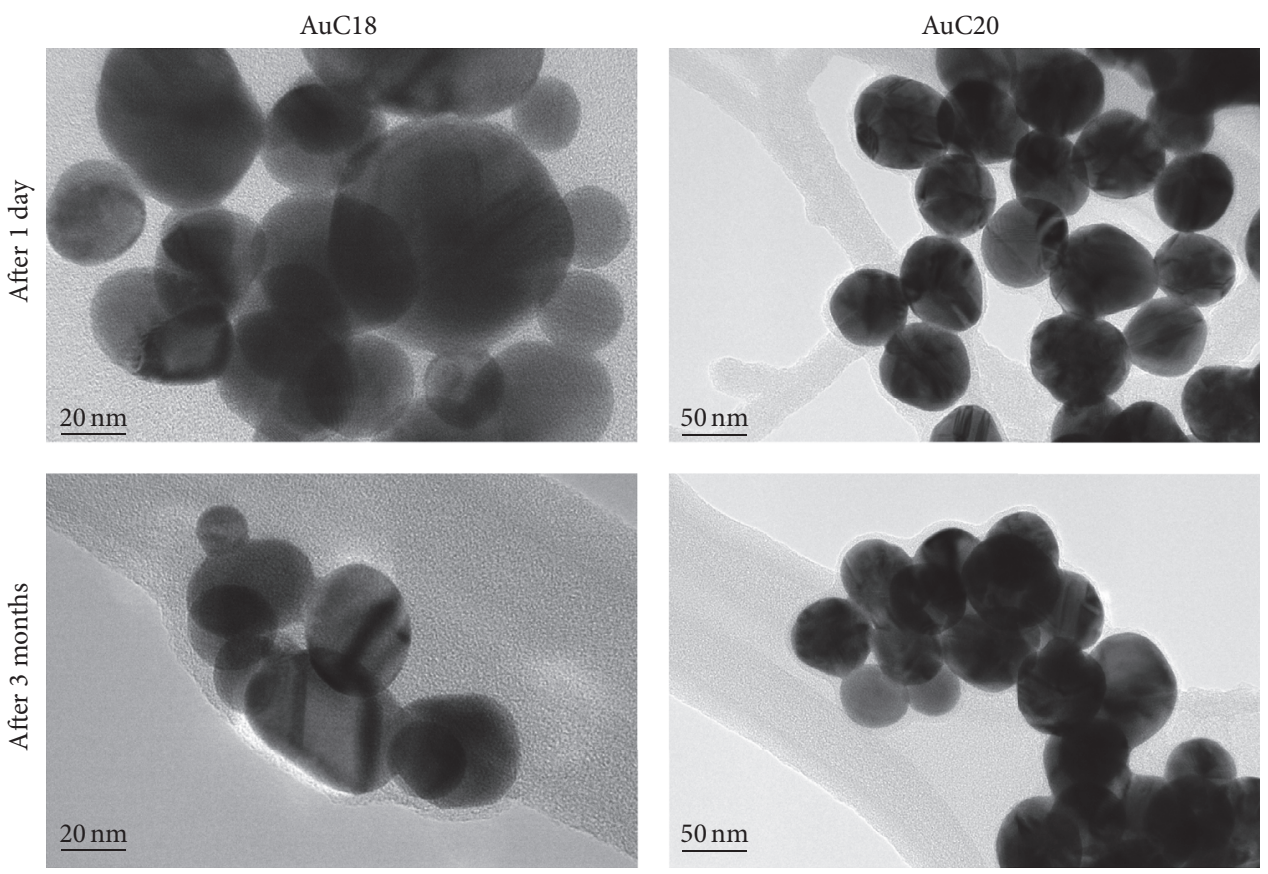

(b)

Figure 2: Size distribution and morphology of AuC18 and AuC20 NPs. (a) Size distribution of AuC18 and AuC20 NPs, as determined by measuring the diameter of at least 200 NPs on TEM images, is presented as frequency distribution (size [nm] versus \% of AuNP with a particular size) and as whiskers. (b) Representative TEM images of the AuC18 and AuC20 taken 1 day or 3 months after the synthesis by modular USP.

of NPs/mL was $4.25 \times 10^{11}$ and $2.71 \times 10^{10}$, respectively (Appendix).

Energy Dispersive Spectroscopy (EDS) analysis confirmed a high purity content of 99.9 wt.\% Au (Figure 3(a)). Besides, the electron diffraction analysis of $\mathrm{AuC18}$ and $\mathrm{AuC20}$ samples showed a space group Fm-3m in both types of AuNPs, which corresponds to the face-centred cubic lattice structure present in Au (Figure 3(b)). The surface energies for grain growth of the Au face-centred cubic lattice were in accordance with those previously described [30]: $a_{\{111\}}<$ $a_{\{100\}}<a_{\{110\}}$. No apparent grain orientation was present. There was a high concentration of grain boundaries and twins present in the AuNPs, while there were no visible vacancies or porosities. This suggested that the growth of the AuNPs during USP was heterogeneous.

Considering that hydrodynamic size and surface charge are the most relevant for biological systems, we analyzed $z$-potential and hydrodynamic sizes of the two types of
AuNPs in citrate buffer by using DLS analysis (Figure 4). DLS measurements of AuNPs in citrate buffer suggested that AuC18 NPs had two size peaks (bimodal size distribution) of $9.8 \pm 3.4 \mathrm{~nm}$ (95.3\% of AuNPs based on volume distribution analysis) and $58 \pm 33.6 \mathrm{~nm}$ (4.7\% of AuNPs). In contrast, AuC20 had unimodal size distribution of $32.0 \pm 19.5 \mathrm{~nm}$ (99.7\% of AuNPs) in the same buffer. The zeta-potential of AuC18 and AuC20 AuNPs in citrate buffer was negatively charged at $-23.2 \pm 1.3 \mathrm{mV}$ and $-28.3 \pm 1.7 \mathrm{mV}$, respectively. The negative zeta-potential of AuNPs most probably helped in the stability and high dispersity of these AuNPs due to repulsive forces, since the hydrodynamic size of AuNPs also did not change significantly more than three months after their initial preparation (data not shown).

Although DLS measurements were in general agreement with the size measured by TEM, some discrepancies between the expected and the obtained values were probably a consequence of large variability in AuNPs' size combined with the 


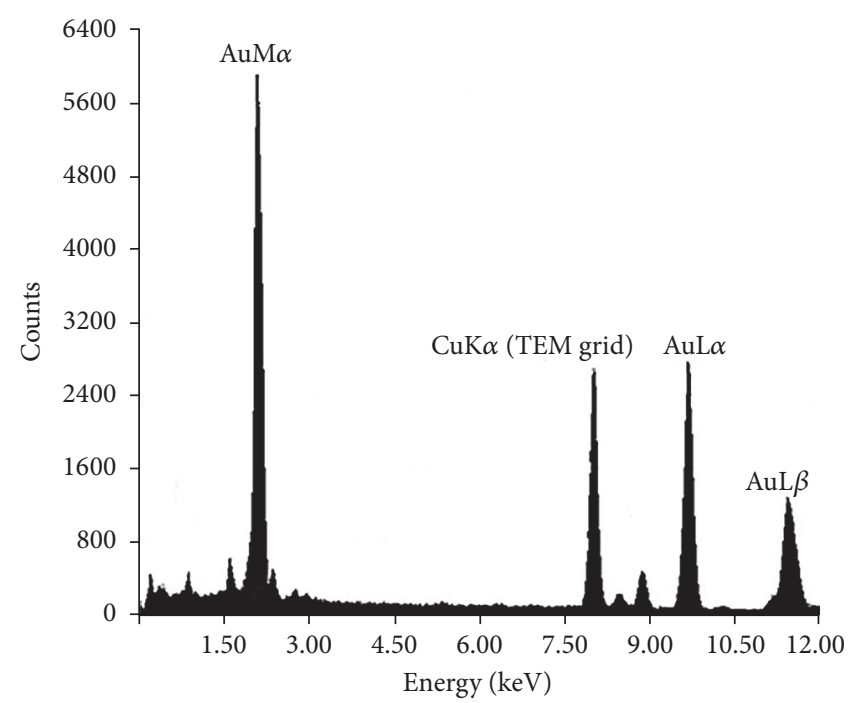

(a)
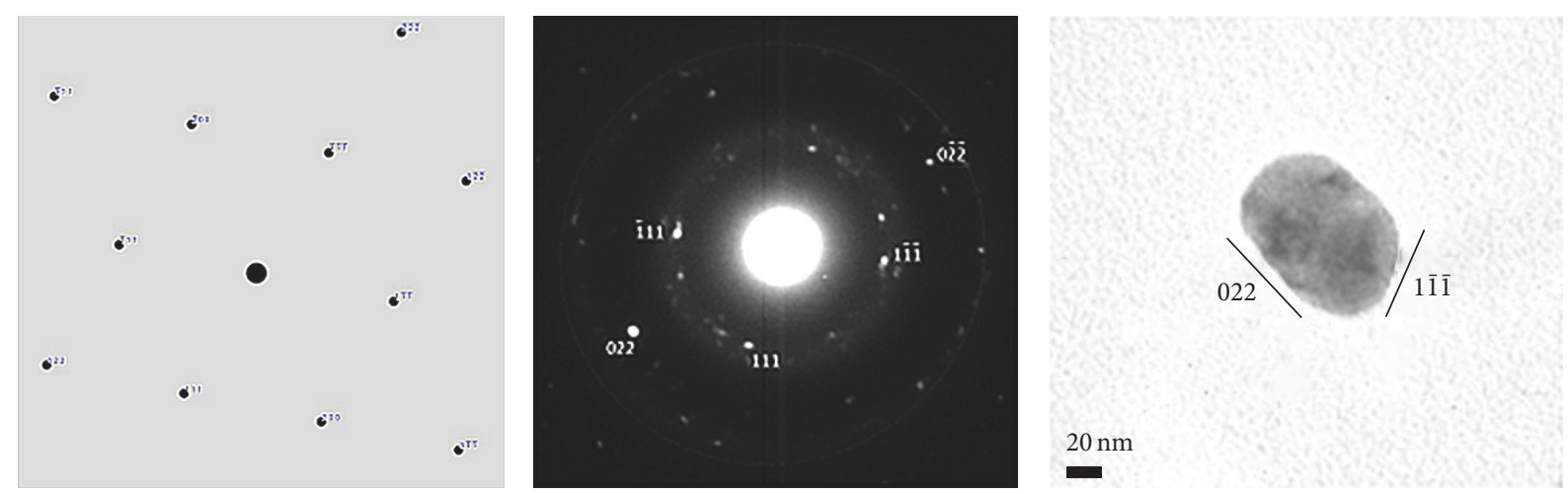

(b)

Figure 3: Composition and crystal structure of AuNPs. (a) Corresponding EDX analysis of the obtained AuNPs is shown. (b) Electron diffraction of a single AuNP with crystal lattice simulation having space group Fm-3m structure and TEM micrograph (lower right) of a single AuNP with visible selected site of electron diffraction.

small sample size obtained during the TEM measurements. On the other hand, DLS sizing of NPs also has certain limitations, especially when the solution contains larger NPs due to their intense light scattering properties [31]. However, both sizing methods indicated that although AuNPs generated by modular USP had a lower size variability compared to standard USP [21], the process needs additional improvements for better size control. The bimodal size distribution of $\mathrm{AuC18}$ was probably a consequence of two mechanisms of their generation during USP: the Gas-to-Particle (GTP) and Droplet-to-Particle (DTP) mechanisms. We showed previously that the GTP mechanism results in the synthesis of much smaller nanoparticles and DTP results in larger nanoparticles [21]. According to the results obtained in this study, the GTP mechanism was most probably predominant, as compared to the DTP for the sample AuC18, which resulted in the synthesis of a larger number of small sized AuNPs. On the other hand, the formation of the AuNPs in sample AuC20 was most probably due to GTP only, which yielded unimodal size distribution in the sample. The reason behind this mechanism is most probably the higher gas flow rates of nitrogen and hydrogen. In high gas flows rates, more turbulence in the system is expected, causing more collisions of aerosol droplets, precursor vapors, and nanoparticles. An increased number of eddies in the gas flow probably caused the increase of nanoparticle sizes with unimodal size distribution. Besides the better size control, the modular USP will require an increase of AuNPs' yield. Namely, taking into account the initial precursor concentration, the amount of precursor solution consumed, and the concentration of AuNPs collected in the final volume, the yields for AuC18 and AuC20 samples were $4.67 \%$ and $6.83 \%$, respectively. The low yield of AuNPs by this USP equipment most probably occurred due to the losses of deposition of AuNPs in the transport tubes, impaction, gravitational sedimentation in the transport tubes and diffusion onto the transport tube walls, turbulence, and thermophoresis.

3.2. Properties of USP-Generated AuNPs in Cell Culture Medium. Besides AuNPs size and surface charge, the optical properties of AuNPs are the most relevant for their biomedical application [1]. In contrast to the acidic environment of 

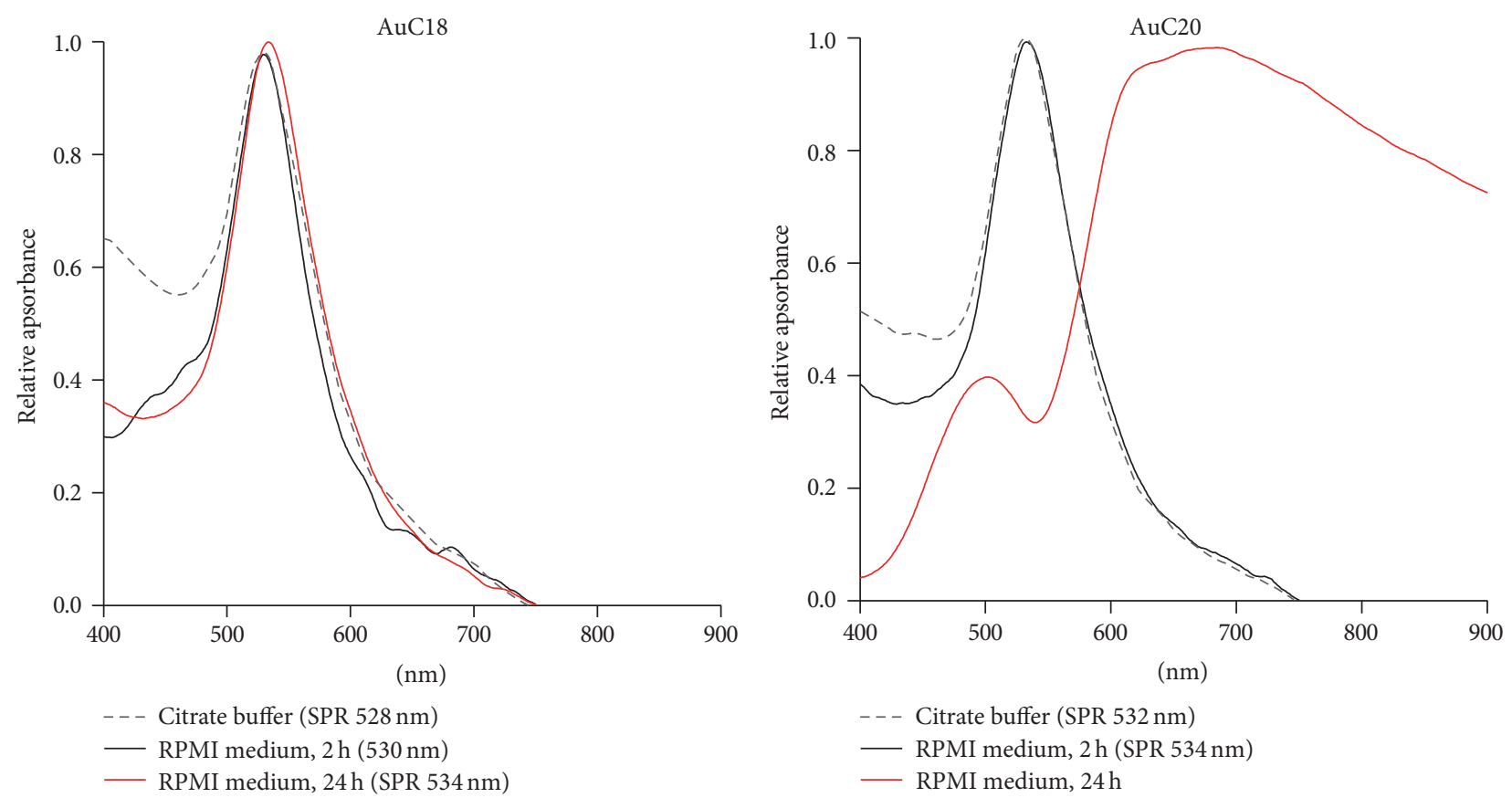

(a)
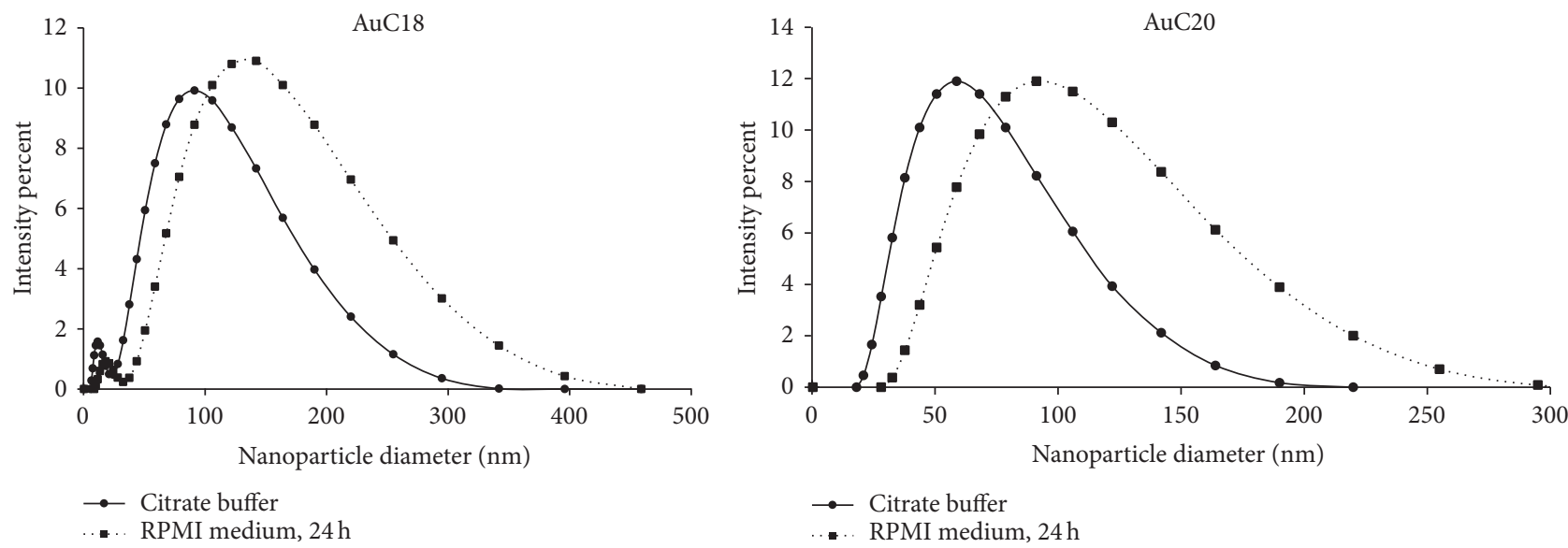

(b)

FIgURE 4: Surface Plasmon Resonance properties and hydrodynamic size distribution of AuNPs in citrate solution and complete RPMI medium. (a) The UV-vis spectra of AuNPs are shown, dispersed either in citrate-solution or in complete RPMI medium for $2 \mathrm{~h}$ or $24 \mathrm{~h}$. (b) The hydrodynamic size of AuNPs was measured either in citrate buffer or in complete RPMI medium by Dynamic Light Scattering.

AuNPs storage (Na-citrate, $\mathrm{pH} 3.5 \pm 0.1$ ), biological fluids are $\mathrm{pH}$ neutral and contain different proteins and ions. Barreto et al. [32] showed recently by using UV-vis spectrometry that citrate-capped AuNPs of different sizes prepared by chemical synthesis were unstable in different ionic strength solutions, including cell culture media, displaying a significant increase in size after conditioning due to agglomeration/aggregation. To examine whether USP-generated AuNPs behave similarly, the UV-vis spectra of AuC18 and AuC20 in citrate solution and after their incubation in the complete RPMI medium for $2 \mathrm{~h}$ or $24 \mathrm{~h}$ were analyzed (Figure 4(a)). AuC18 NPs had the SPR peak at $528 \mathrm{~nm}$ in citrate solution. A red shift of $2 \mathrm{~nm}$ was observed after their conditioning in the complete RPMI medium for $2 \mathrm{~h}$. A further increase in SPR peak was observed after $24 \mathrm{~h}$ of conditioning by a total of $6 \mathrm{~nm}$. AuC20 NPs had the SPR peak at $532 \mathrm{~nm}$ in the citrate solution due to their larger average size compared to AuC18 NPs. A similar red shift of $2 \mathrm{~nm}$ was observed after the incubation of AuC20 in the complete RPMI medium for $2 \mathrm{~h}$. However, after $24 \mathrm{~h}$ of conditioning, a loss of SPR band and a strong red shift absorbance were observed. The detected SPR peaks in citrate solution were probably a result of optical activity of all NPs present in the solution, which could explain their higher SPR values as compared with NPs with a small size distribution. The $2 \mathrm{~nm}$ red shifts in the SPR peaks after shortterm conditioning could be explained by the change of local refraction index at the surface of AuNPs due to adsorption of medium components [3-5]. In contrast, larger red shifts and 
a loss of the SPR band could indicate their increase in size due to agglomeration [32].

To determine how the results from UV-vis analysis relate to the change in AuNPs' hydrodynamic size after conditioning, DLS measurements of AuNPs conditioned for $24 \mathrm{~h}$ in complete medium were carried out in comparison to DLS size in citrate buffer. The size distribution of sample $\mathrm{AuC18}$ after the conditioning increased 1.5-2 times (size after conditioning was $15.7 \pm 4.9 \mathrm{~nm}$ ( $95 \%$ of AuNP) and $99.9 \pm 62.5 \mathrm{~nm}$ ( $5 \%$ of AuNPs)), as compared to AuC18 size in Na-citrate solution, which could explain the $6 \mathrm{~nm}$ shift in the SPR peak. In the case of the AuC20 samples, the size distribution values increased up to 3 times (size after conditioning was $102.3 \pm 44.4 \mathrm{~nm}$ ) (Figure 4(b)). This corresponds to the changes observed in the SPR of AuC20, as NPs larger than $100 \mathrm{~nm}$ do not have a clearly defined SPR band within 500-600 nm due to the dominant contributions from higher-order electron oscillations [33]. The aggregation of AuNPs in the complete RPMI medium was not immediate, most probably due to adsorption of serum proteins on their surface. In line with this, it was shown that aggregation/agglomeration of chemically synthesized citrate-capped AuNPs occurs immediately upon placing them in artificial saliva or serum-free cell culture media (RPMI or DMEM), whereas the agglomeration in the serumcontaining media was much slower [32, 34, 35]. These studies suggested that agglomeration/aggregation of citrate-capped AuNPs results from changes on the extension of capping by the citrate anions at the surface [36]. Although the increase in hydrodynamic size of USP-generated AuNPs in biological media was much smaller compared to those described for the chemically synthesized citrate-AuNPs, the expected size-dependent changes in the optical properties of USPgenerated AuC20 NPs due to agglomeration/aggregation do not allow their easy application in diagnostics. However, the size of both AuNPs in medium was still suitable for potential development of drug-delivery systems. Therefore, we next investigated the cytocompatibility of AuNPs on L929 cells, which are recommended by the ISO 10993-5 Standard, and primary human monocytes to examine their potential immunomodulatory properties.

3.3. Cytocompatibility of AuNPs. Our previous studies suggested that pure AuNPs produced by USP are not cytotoxic for L929 cells, rat thymocytes, and splenocytes up to $100 \mu \mathrm{g} / \mathrm{mL}[16,17]$. Furthermore, the improvement of USP by incorporation of the preheating chamber does not compromise the good biocompatibility of these AuNPs [21]. However, the biocompatibility of citrate-capped AuNPs is still controversial [23-25, 37]. Therefore, we evaluated whether citrate-stabilized AuNPs produced by modular USP induce the cytotoxicity of L929 cells after $24 \mathrm{~h}$ and whether they affect the proliferation of these cells after 3 days of cultivation.

Although the DLS spectra showed a large variation in the size of AuNPs and their increase upon interaction with medium, L929 clearly internalized AuNPs after $24 \mathrm{~h}$ of incubation (Figure 5(a)). Considering that AuNPs did not agglomerate immediately upon their interaction with medium and that the internalization of AuNPs is a relatively quick process [38], it is possible that the majority of AuNPs were internalized by L929 cells in their native size rather than as agglomerates. Besides, it is not known whether L929 cells internalize monodispersed or agglomerated AuNPs at a higher rate as is known for other cell types [39].

To analyze the cytocompatibility of AuNPs in L929 cell culture, viability, apoptosis, metabolic activity, and proliferation dynamics were analyzed. The viability assay, based on Trypan blue staining, showed no signs of cytotoxicity of AuC18 and AuC20 in L929 cell culture up to $200 \mu \mathrm{g} / \mathrm{mL}$ (Figure 5(b)). Similar results were obtained by Annexin-V/PI staining of cells after the culture, as only a mild increase in early and late apoptotic cells (Annexin-V+/PI- and Annexin$\mathrm{V}+/ \mathrm{PI}+$ ) was observed in the cultures with $200 \mu \mathrm{g} / \mathrm{mL}$ of AuNPs, whereas lower concentrations had no cytotoxic effects (Figure 5(c)). Additionally, the MTT assay carried out to determine the total metabolic activity of L929 cell cultures in the presence of AuNPs also suggested that AuNPs prepared by USP do not affect the metabolic activity in the culture at the concentrations lower than $200 \mu \mathrm{g} / \mathrm{mL}$ (Figure 5(d)). The dose-dependent effect of AuC18 and AuC20 NPs on proliferation of L929 cells was carried out using the $3 \mathrm{H}$-thymidine incorporation assay as the most sensitive method for the cell proliferation studies (Figure 5(b)). The results showed that both types of nanoparticles inhibited the proliferation of L929 cells at a concentration of $100 \mu \mathrm{g} / \mathrm{mL}$ and higher. Such an effect was similar to the one we observed previously, in which nonstabilized AuNPs prepared by modular USP did not inhibit the proliferation of L929 cells at the concentrations lower than $100 \mu \mathrm{g} / \mathrm{mL}$ [21]. Considering that $100 \mu \mathrm{g} / \mathrm{mL}$ of USP-generated AuNPs was not toxic for L929, the observed antiproliferative effect could be explained by the fact that AuNPs can affect the cytoskeleton upon their internalization and impair the cellular processes necessary for proliferation $[24,25]$. However, this hypothesis needs to be tested independently for the USP-generated AuNPs.

3.4. Anti-Inflammatory Properties of AuNPs. Previously, we showed that AuNPs prepared by USP [16] and those synthesized chemically [26] can induce direct immunomodulatory effects when applied at nontoxic concentration $(50 \mu \mathrm{g} / \mathrm{mL})$ predominantly by modulating the functions of APCs. Considering these results, as well as those obtained in this study, we chose the concentration of $50 \mu \mathrm{g} / \mathrm{mL}$ to investigate the immunomodulatory/anti-inflammatory properties of $\mathrm{AuC18}$ and AuC20 nanoparticles, using primary human monocytes as a model system. Monocytes represent the major population of phagocytic APCs [27], but the data on the effects of citratecapped AuNPs on human monocytes are quite scarce.

Therefore, the monocytes were cultivated with $\mathrm{AuCl} 8$ or $\mathrm{AuC} 20$ for $24 \mathrm{~h}$, in either the presence or absence of LPS, followed by assessment of the monocytes' viability and phenotypic and functional properties. After $24 \mathrm{~h}$ cultures, monocytes internalized AuNPs, as judged by phase contrast microscopy (Figure 6(a)) and flow cytometry (Figure 6(b)). These results suggested that AuNPs, irrespective of their large size variability, were internalized easily by monocytes, most probably as provisionally stabilized by serum proteins. Upon the internalization, AuC18 and AuC20 did not induce 

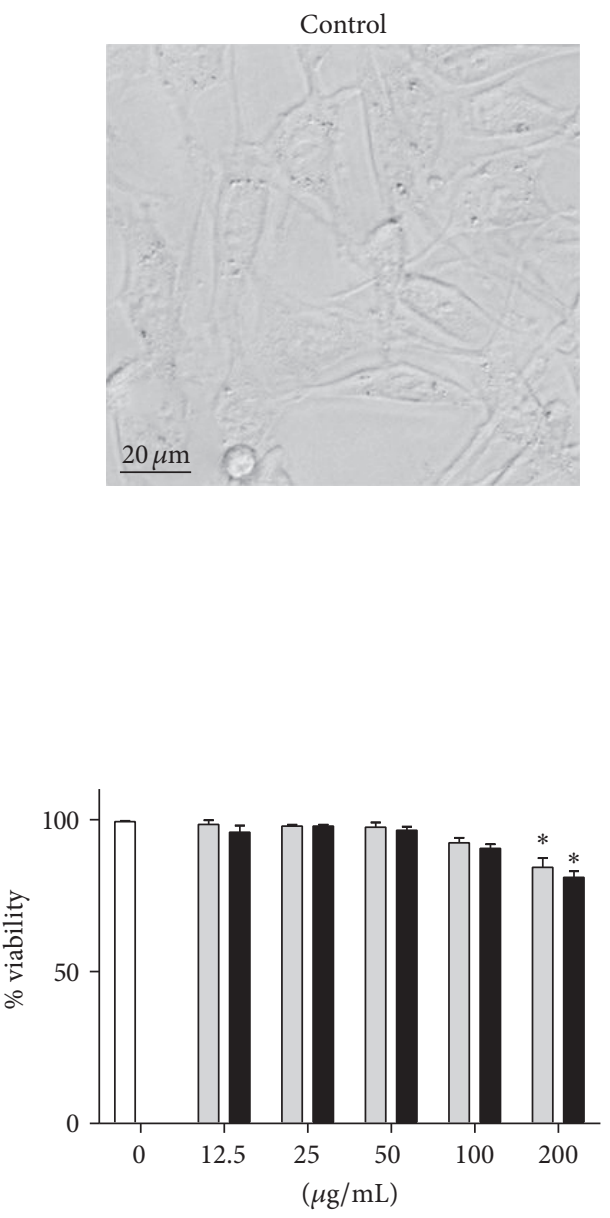

$\mathrm{AuC18}$
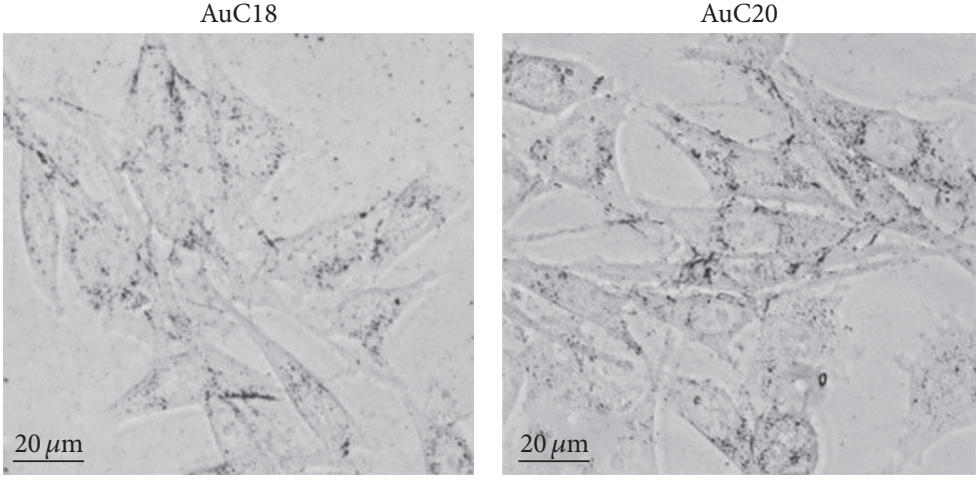

(a)
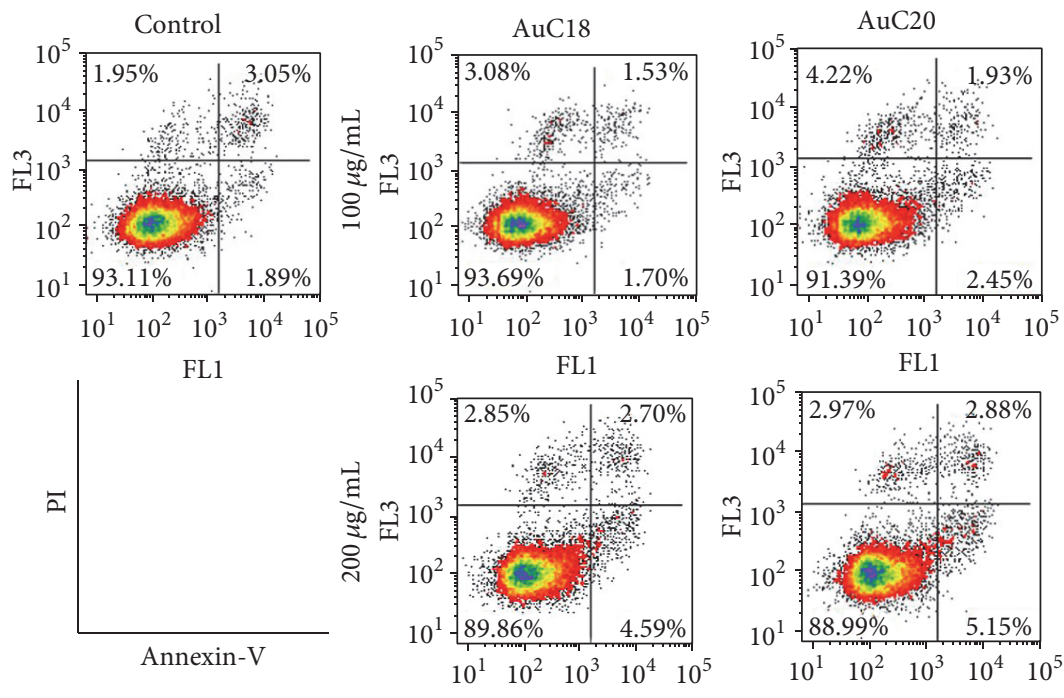

FL1

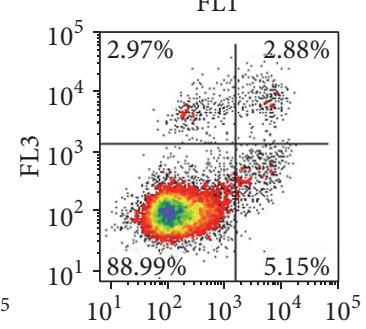

(c)

(b)

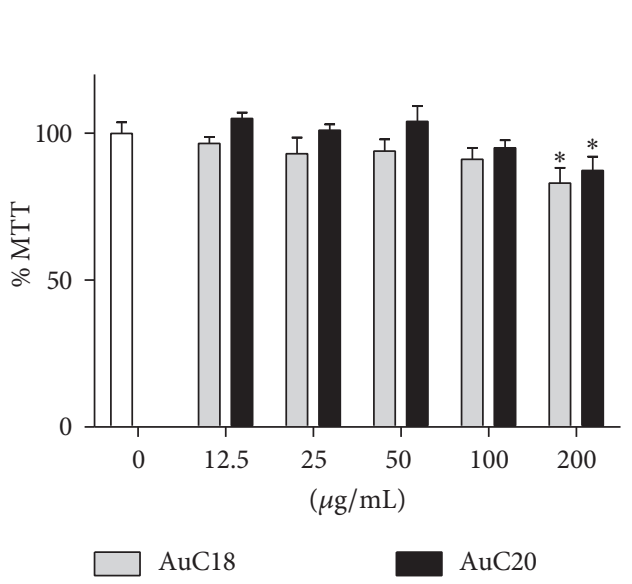

(d)

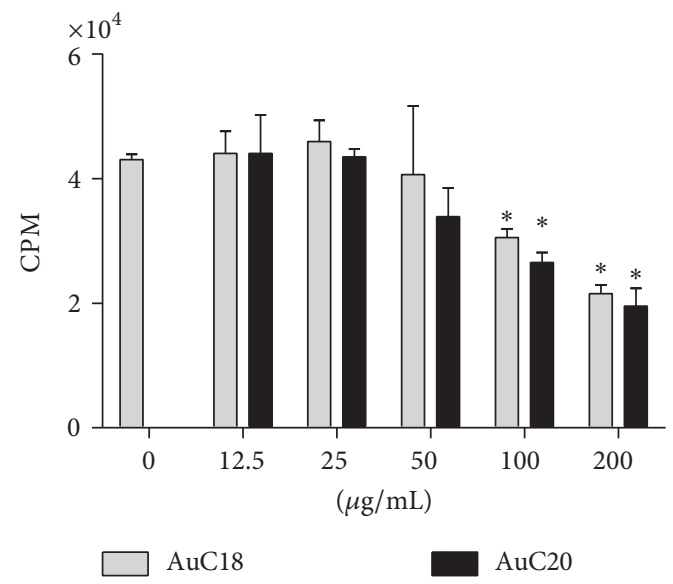

(e)

Figure 5: Cytocompatibility of AuC18 and AuC20 in culture with L929 cells. (a) Phase contrast microscopy of L929 cells cultivated with AuNPs $100 \mu \mathrm{g} / \mathrm{mL}$ for $24 \mathrm{~h}$ is shown. (b) The viability of cells cultivated with AuNPs $(12.5-200 \mu \mathrm{g} / \mathrm{mL})$ is shown, as determined by the Trypan blue exclusion test. The results obtained in each experiment were normalized to control (100\%), and these are shown as mean viability \pm SD ( $n=3$ independent experiments). (c) A representative experiment is shown on the assessment of apoptosis of L929 cells cultivated with AuNPs by Annexin-V/PI staining out of three experiments with similar results. (d) Relative metabolic activity of L929 cells cultivated with AuNPs $(12.5-200 \mu \mathrm{g} / \mathrm{mL})$ is shown, as determined by MTT assay. The results are presented as mean $\% \mathrm{MTT} \pm \mathrm{SD}(n=3$ independent experiments) relative to control (100\%). (e) Proliferation of L929 cells in the presence of AuNPs $(12.5-200 \mu \mathrm{g} / \mathrm{mL})$ was determined after 3-day cultures, by $3 \mathrm{H}$-thymidine incorporation assay. The results are shown as mean CPM (counts per minute) $\pm \mathrm{SD}$ ( $n=3$ experiments). ${ }^{*} p<0.05$ compared to control. 

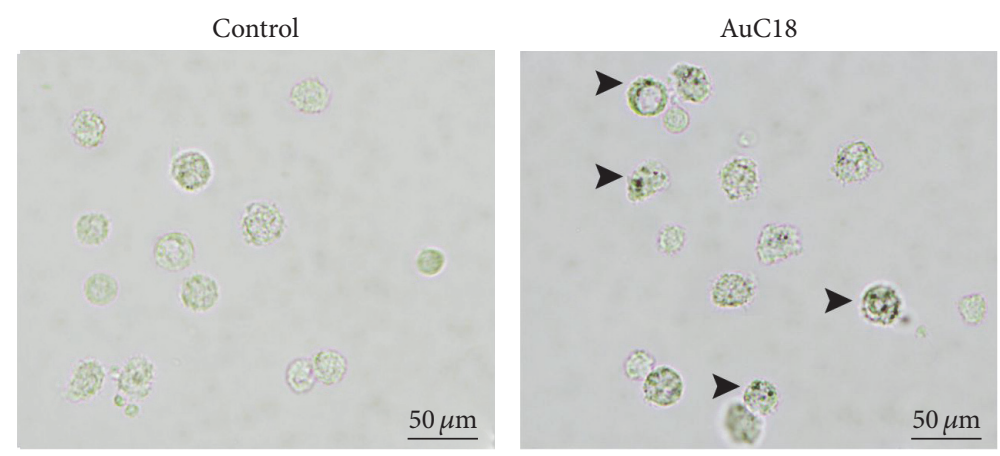

(a)
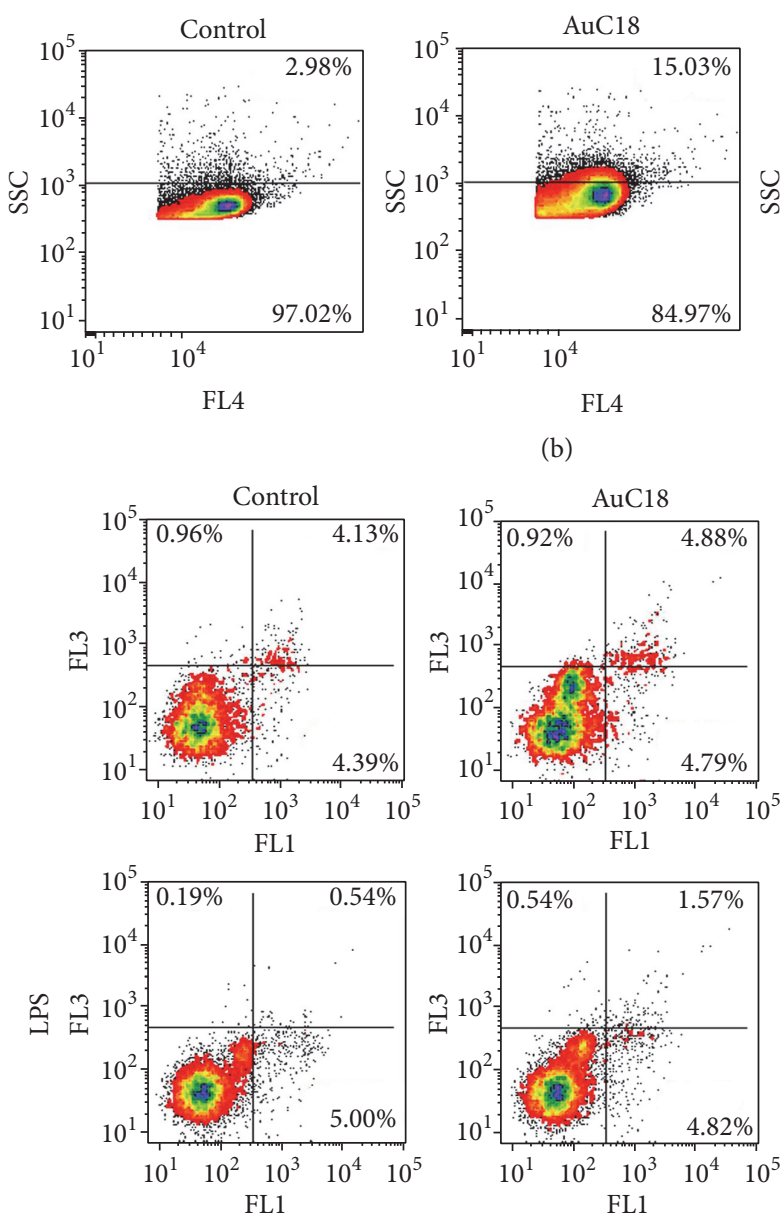

(d) (b)

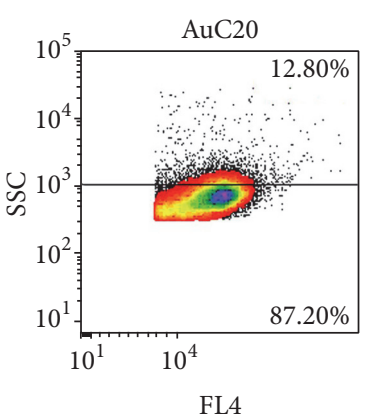

FL4
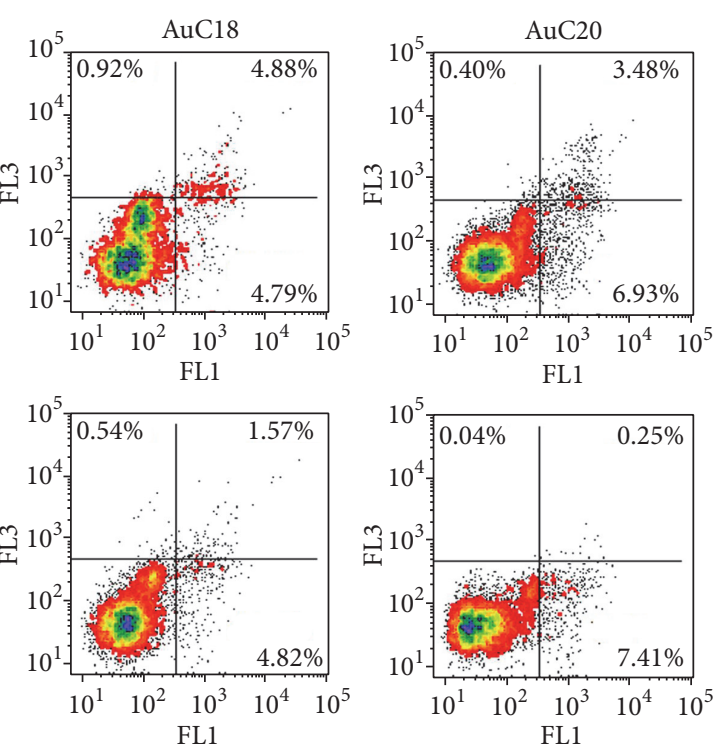

FL
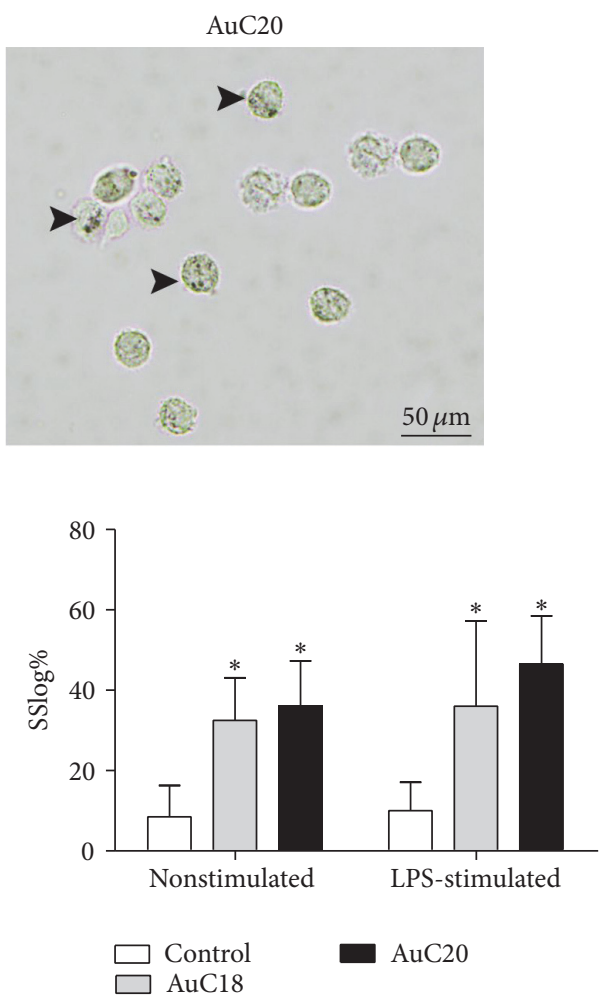

(c)

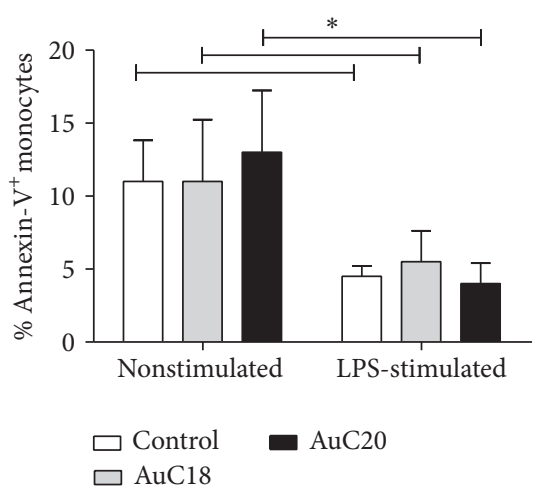

(e)

FIgURE 6: Internalization of AuC18 and AuC20 NPs by monocytes and their viability. (a) Representative images of monocytes cultivated in the presence or absence of AuNPs $(50 \mu \mathrm{g} / \mathrm{mL})$ for $24 \mathrm{~h}$ are shown. The black arrows point to the cells with accumulated AuNPs. (b) A representative flow cytometry analysis of internalization of AuNPs by monocytes is shown, in which the side scatter parameter (SSlog) was used as an indicator of internal complexity of the cells. (c) Summarized results obtained from such analyses are shown as mean SSlog\% \pm SD of 3 independent experiments. ${ }^{*} p<0.05$ compared to nontreated monocytes (d) Representative analysis of apoptosis of LPS-treated and nontreated monocytes cultivated with AuNPs for $24 \mathrm{~h}$ and then stained with Annexin-V-FITC and PI. (e) Summarized data on total Annexin $-\mathrm{V}^{+}$expression is presented as mean $\% \pm \mathrm{SD}$ of 3 independent experiments. ${ }^{*} p<0.05$ compared as indicated. 

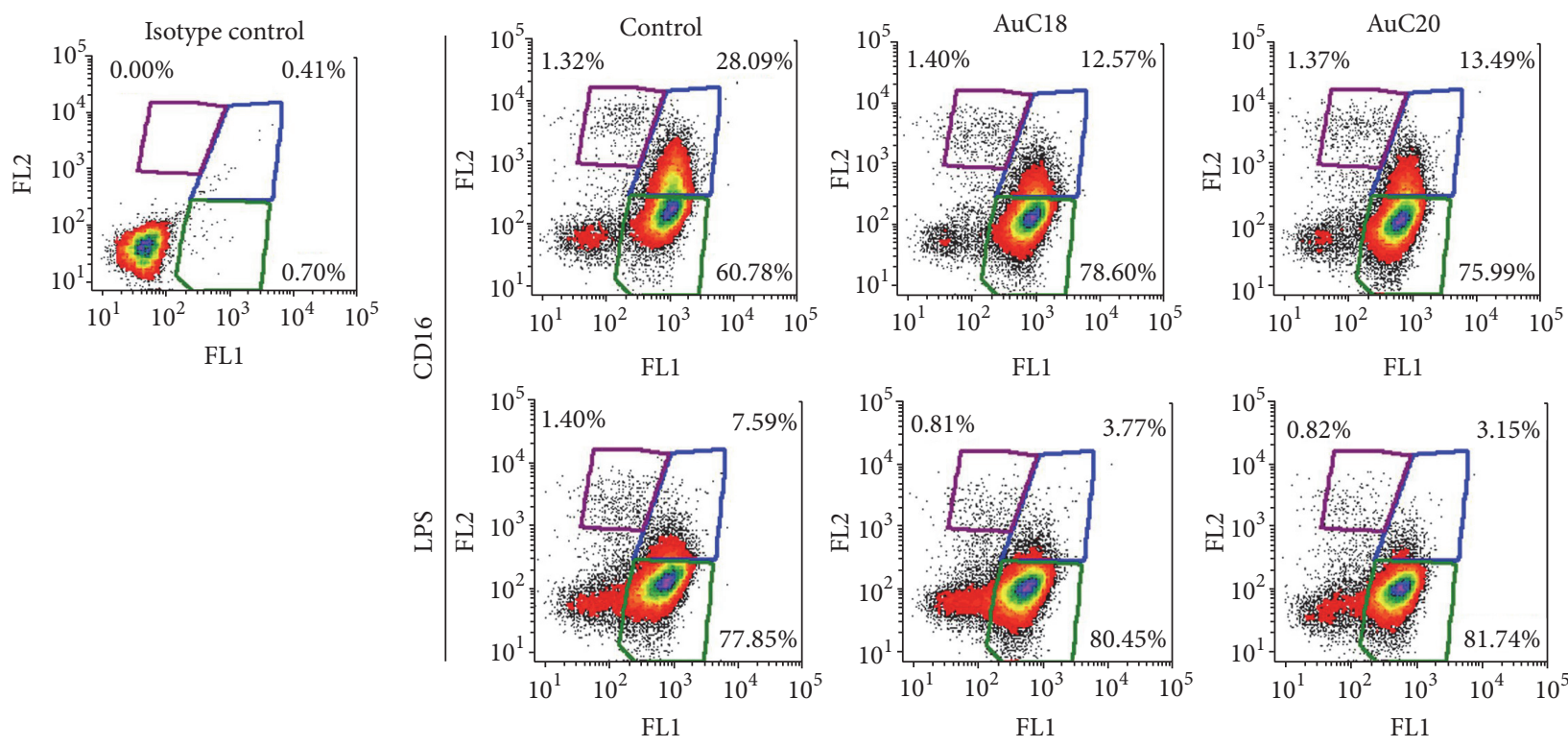

CD14

(a)
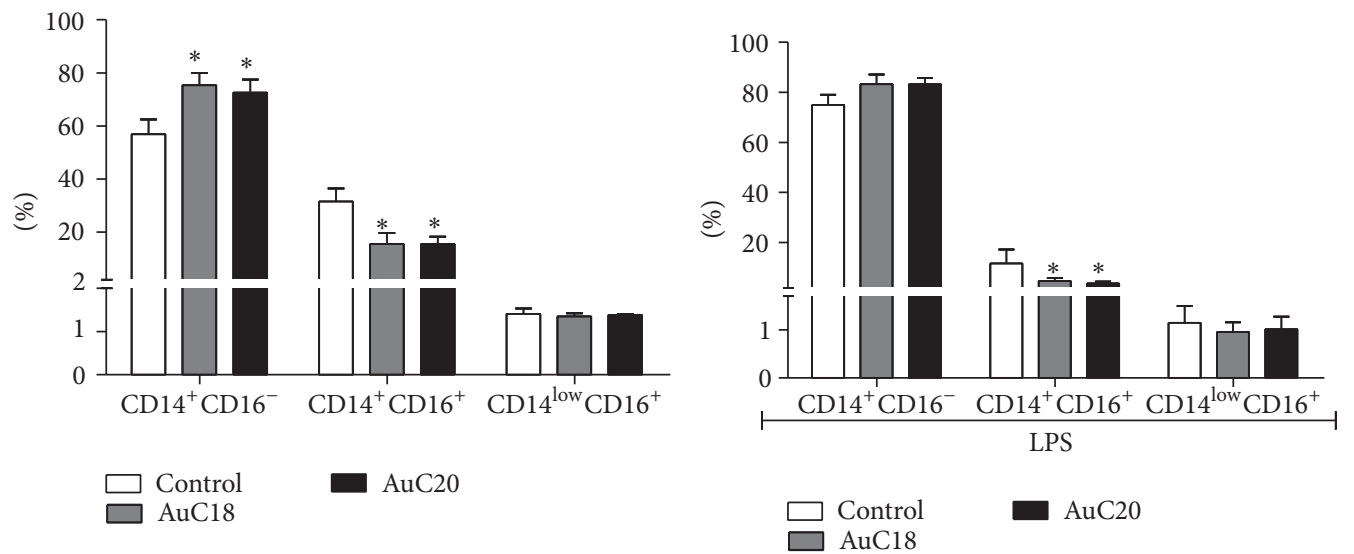

(b)

Figure 7: Effect of AuC18 and AuC20 on monocytes subpopulations. (a) Representative dot plots of monocytes stained on CD14-FITC and CD16-PE after $24 \mathrm{~h}$ cultures in the presence or absence of LPS and AuC18 and AuC20 NP as indicated. (b) Summarized results are shown as mean $\% \pm \mathrm{SD}(n=3)$ (two-way ANOVA). ${ }^{*} p<0.05$ compared to control.

significant apoptosis of monocytes compared to the control, as judged by Annexin-V and PI staining (Figures 6(c) and 6(d)). The apoptosis of monocytes in the presence of LPS was significantly lower, as expected from previous findings [40], and AuNPs did not modify significantly the prosurvival effect of LPS on monocytes. These results are in line with the recent finding of Chhour et al. [41], showing that AuNPs stabilized with different capping agents do not cause the apoptosis of the mouse monocyte cell line RAW 264.7 after internalization, even when applied in much higher concentrations. The low toxicity of citrate-capped AuNPs was also shown in experiments with different cell lines [42]. To our knowledge, this is the first finding indicating that good cytocompatibility of citrate-capped AuNPs could be expected for primary human monocytes as well.
Peripheral blood monocytes constitute two functionally distinct subpopulations, classical $\mathrm{CD}^{+}{ }^{+}$(which could be subdivided further into $\mathrm{CD} 14^{+} \mathrm{CD} 16^{-}$and $\mathrm{CD} 14^{+} \mathrm{CD} 16^{+}$) and $\mathrm{CD} 14^{\text {low }} \mathrm{CD}^{+} 6^{+}$monocytes, and the changes in these subpopulation ratios were shown to correlate with the inflammatory response of the host $[43,44]$. However, the analysis of monocytes subpopulations upon their interaction with AuNPs has not been carried out so far. To observe whether AuNPs affect the distribution of these subpopulations, the monocytes cultivated with AuC18 and AuC20 nanoparticles, in either the presence or absence of LPS, were analyzed by flow cytometry. The results suggested that the population of analyzed monocytes contained more than $83 \%$ of $\mathrm{CD} 14^{+}$ cells, and neither AuNPs nor LPS affected the expression of this molecule (Figure 7). However, both types of AuNPs 
downregulated the expression of $\mathrm{CD} 16$ on $\mathrm{CD} 14^{+}$nonstimulated monocytes significantly, thus reducing the ratio of $\mathrm{CD}_{14}{ }^{+} \mathrm{CD} 16^{+}$and $\mathrm{CD} 14^{+} \mathrm{CD} 16^{-}$subpopulations. Thereby, the percentage of $\mathrm{CD} 14^{\text {low }} \mathrm{CD} 16^{+}$population remained relatively constant. LPS reduced the expression of $\mathrm{CD} 16$ on $\mathrm{CD} 14^{+}$ monocytes significantly. Still, AuNPs reduced additionally the expression of $\mathrm{CD} 16$ on $\mathrm{CD} 14^{+}$monocytes stimulated with LPS, without affecting the expression of $\mathrm{CD} 16$ on $\mathrm{CD} 14^{\text {low }}$ subpopulation significantly.

$\mathrm{CD} 4^{+} \mathrm{CD} 16^{-}$and $\mathrm{CD} 14^{+} \mathrm{CD} 16^{+}$monocytes were shown to have different chemokine receptors and capacities to secrete cytokines upon in vitro stimulation [45]. In line with this, the $\mathrm{CD} 14^{+} \mathrm{CD} 16^{+}$population is considered a proinflammatory type which is increased during different infections $[46,47]$. They secrete high levels of TNF- $\alpha$ upon TLR2 and TLR4 ligation [48], and they give rise to inflammatory DCs $[49,50]$. Therefore, the effect of $\mathrm{AuC1} 8$ and AuC20 on shifting the ratio of these two populations toward a $\mathrm{CD} 14^{+} \mathrm{CD} 16^{-}$ subpopulation could be interpreted as an anti-inflammatory effect. In contrast to $\mathrm{CD} 14^{+}$monocytes, which extravasate into peripheral tissue to give rise to macrophages and DCs, $\mathrm{CD} 14^{\text {low }} \mathrm{CD} 16^{+}$monocytes are limited to blood vessel lumen and patrol the endothelial surface for potential damage and coordinate its repair by recruiting neutrophils as required $[51,52]$. Therefore, it is of crucial importance that this population of monocytes was not affected by AuNPs during the downregulation of $\mathrm{CD} 16$ on $\mathrm{CD}_{14}{ }^{+}$cells. Furthermore, these results also suggest that the effects are not mediated directly through CD16 downregulation but rather through modulation of the inflammatory monocytes type.

To assess whether AuNPs really possess anti-inflammatory effects on monocytes, the expression of key molecules responsible for antigen presentation and costimulation (HLA-DR and CD86, resp.) and proinflammatory cytokines TNF- $\alpha$ and p40 (a common subunit of IL-12 and IL23) [53] were assessed in monocytes cultivated with AuNPs in the presence or absence of LPS for $24 \mathrm{~h}$ (Figure 8). LPS upregulated the expression of HLA-DR and TNF- $\alpha$ significantly, as expected from previous studies [54]. In contrast, we observed a downregulation of CD86 expression by LPS-treated monocytes, which could be explained by the tolerogenic effect of endotoxin on monocytes after $24 \mathrm{~h}$ stimulation [55]. The expression of p40 in LPS-treated monocytes was not changed significantly in these experiments. Both $\mathrm{AuC18}$ and AuC20 downregulated significantly CD86 expression by both nonstimulated and LPS-stimulated monocytes. AuC18 NPs downregulated the expression of HLA-DR by nonstimulated and LPS-stimulated monocytes, whereas AuC20 had such an effect only on LPS-stimulated monocytes. Additionally, intracellular staining showed that both $\mathrm{p} 40$ and TNF- $\alpha$ were downregulated in LPS-stimulated monocytes treated with both types of AuNPs. AuC18 had the same effects on the examined cytokines in nonstimulated monocytes, whereas AuC20 downregulated significantly the expression of TNF- $\alpha$ in nonstimulated monocytes but not $\mathrm{p} 40$.

Considering that both TNF- $\alpha$ and p 40 are proinflammatory cytokines, these results confirmed that citrate-capped
$\mathrm{AuC} 18$ and $\mathrm{AuC} 20$ produced by modular USP possess antiinflammatory effects. The somewhat stronger effect of $\mathrm{AuCl} 18$ on downregulation of HLA-DR and p40 expression could be explained by the fact that AuC18 contained a much higher frequency of smaller AuNPs, which is in line with our previous results, showing that smaller AuNPs possess stronger immunosuppressive properties $[16,26]$. Recently, citratecapped AuNPs were used as a precursor for 11-MUDA AuNPs which were exploited for noninvasive imaging of monocyte accumulation within atherosclerotic plaques with CT [37]. The authors selected these nanoparticles based on their noncytotoxic and nonmodulatory effects on TNF- $\alpha$ and IL6 production by mice monocytes. However, besides precise imaging, it would be of great benefit for the atherosclerotic patients if such nanoparticles would induce the suppression of inflammation in the plaques. Considering that TNF- $\alpha$, IL-12, and IL-23 are considered proatherogenic [56], the suppression of these cytokines in plaque homing monocytes would be beneficial for the therapy of atherosclerosis. A similar conclusion could be drawn from the results on the proatherogenic role of CD86 and HLA-DR expression by monocytes, which were shown as critical for pathogenic $\mathrm{T}$ cell survival in the presence of oxidized LDL and MHCrestricted $\mathrm{T}$ cell proliferative response to oxLDL [57]. Therefore, downregulation of CD86 and HLA-DR in monocytes by AuNPs could also be considered beneficial for their potential application in the atherosclerosis treatment. In addition, the anti-inflammatory effects of citrate-capped AuNPs derived from UPS could be beneficial for the treatment of other inflammatory and autoimmune conditions as well. However, it should be tested additionally whether similar effects can be induced in vivo, and if so, are there any potentially adverse effects related to the tolerogenic potential of AuNPs?

\section{Conclusions}

We showed for the first time that modular USP, combined with citrate stabilization, enabled the synthesis of highly pure, spherical, nonagglomerated AuNPs, which are stable in citrate solution. However, upon interaction with biological medium, these AuNPs agglomerate/aggregate, which does not provide a good platform for further development of diagnostic tools based on these particles. However, due to their hydrodynamic nanosize, good cytocompatibility, and the ability to enter primary human monocytes and induce anti-inflammatory effects, citrate-capped AuNPs prepared by USP could be beneficial for further development of drug-delivery systems and in the therapy of inflammatory conditions in which phagocytic cells play a key role.

\section{Appendix}

\section{Theoretical Calculation of the Amount of AuNPs}

Assumptions A.1. The AuNPs were dispersed completely and were perfectly spherical and had high purity with uniform density. 

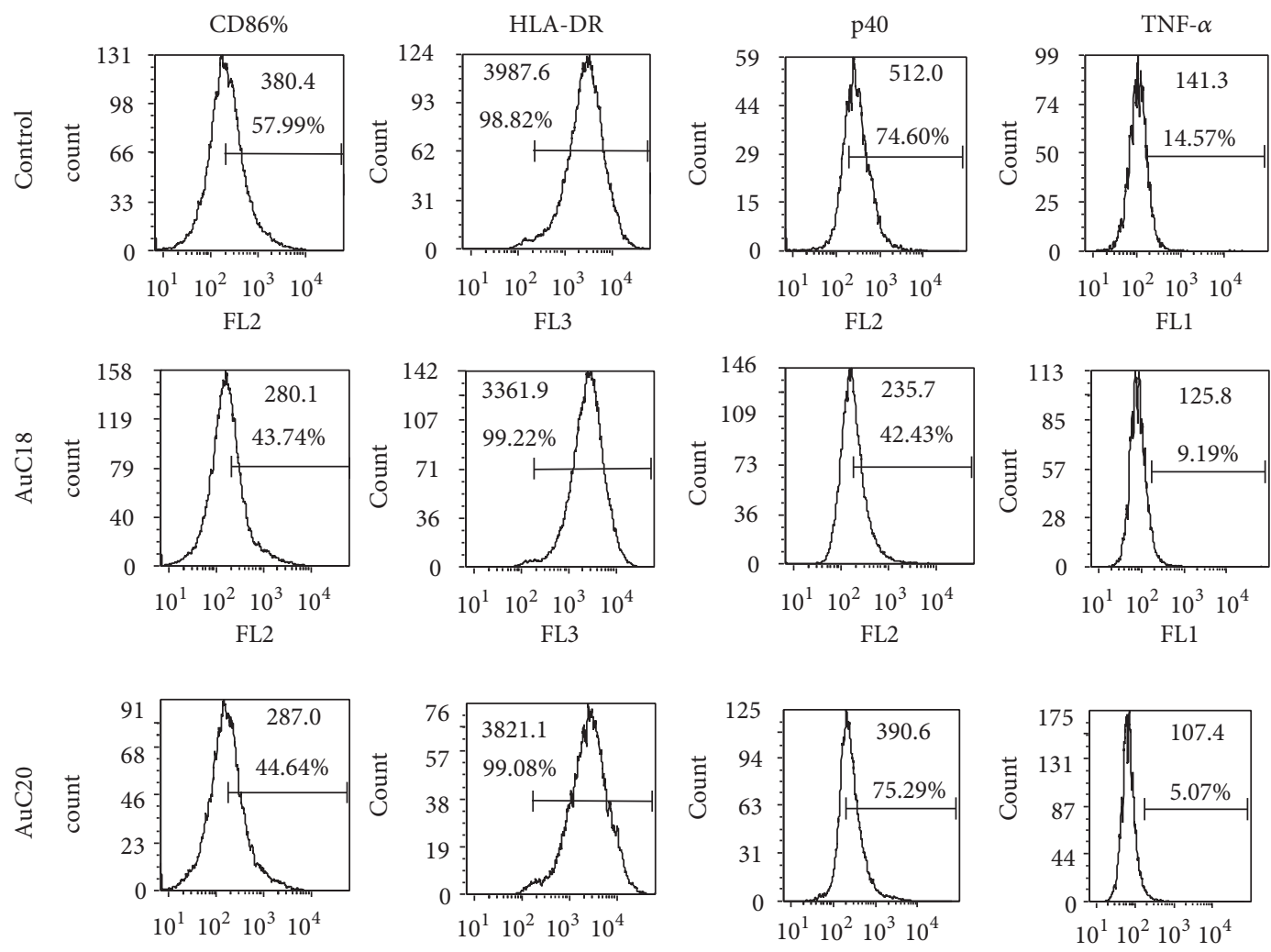

FL3

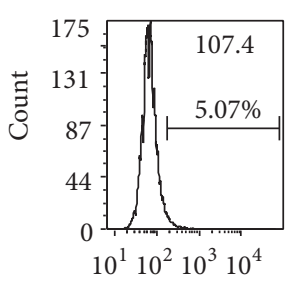

FL2

LPS
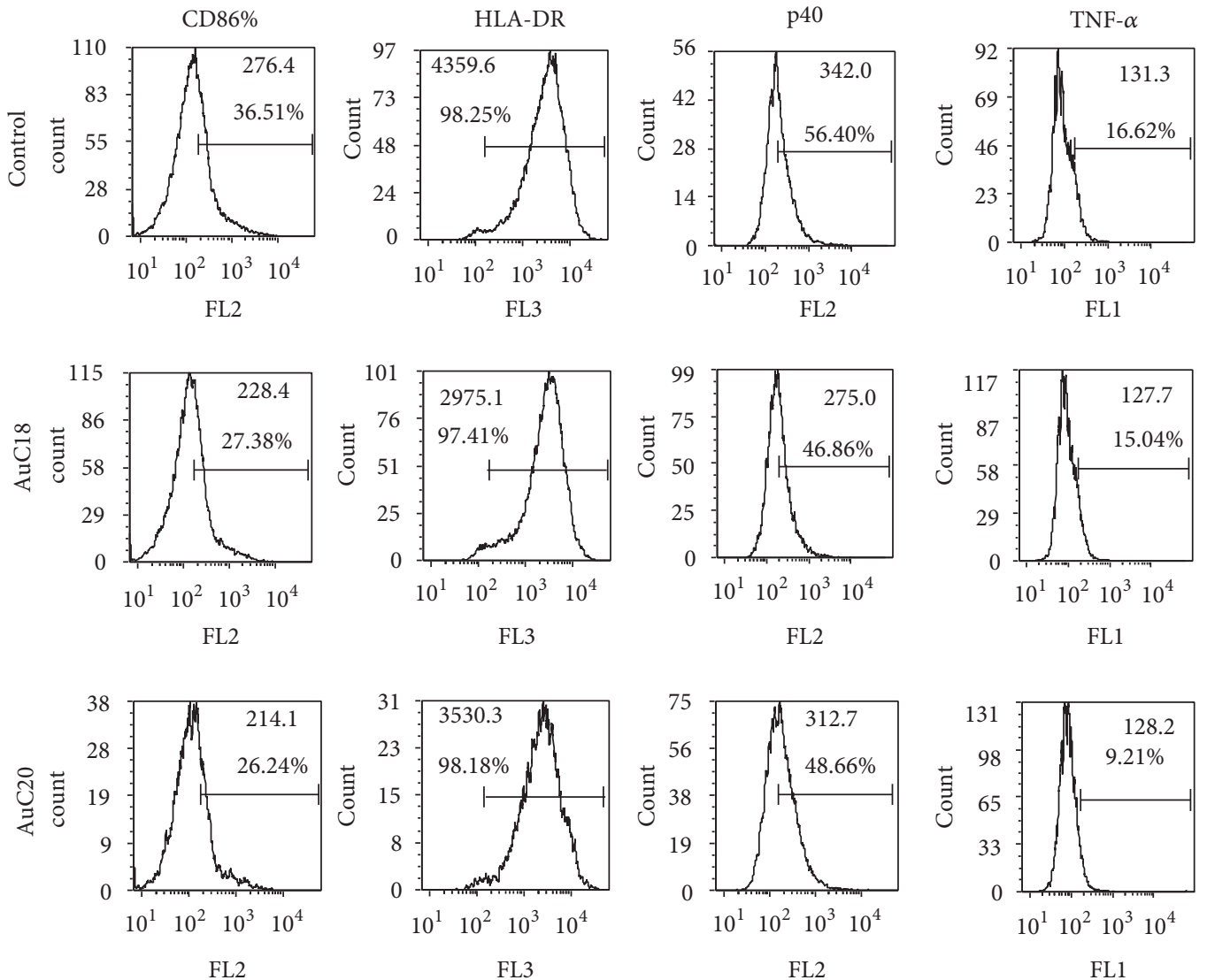

(a)

Figure 8: Continued. 

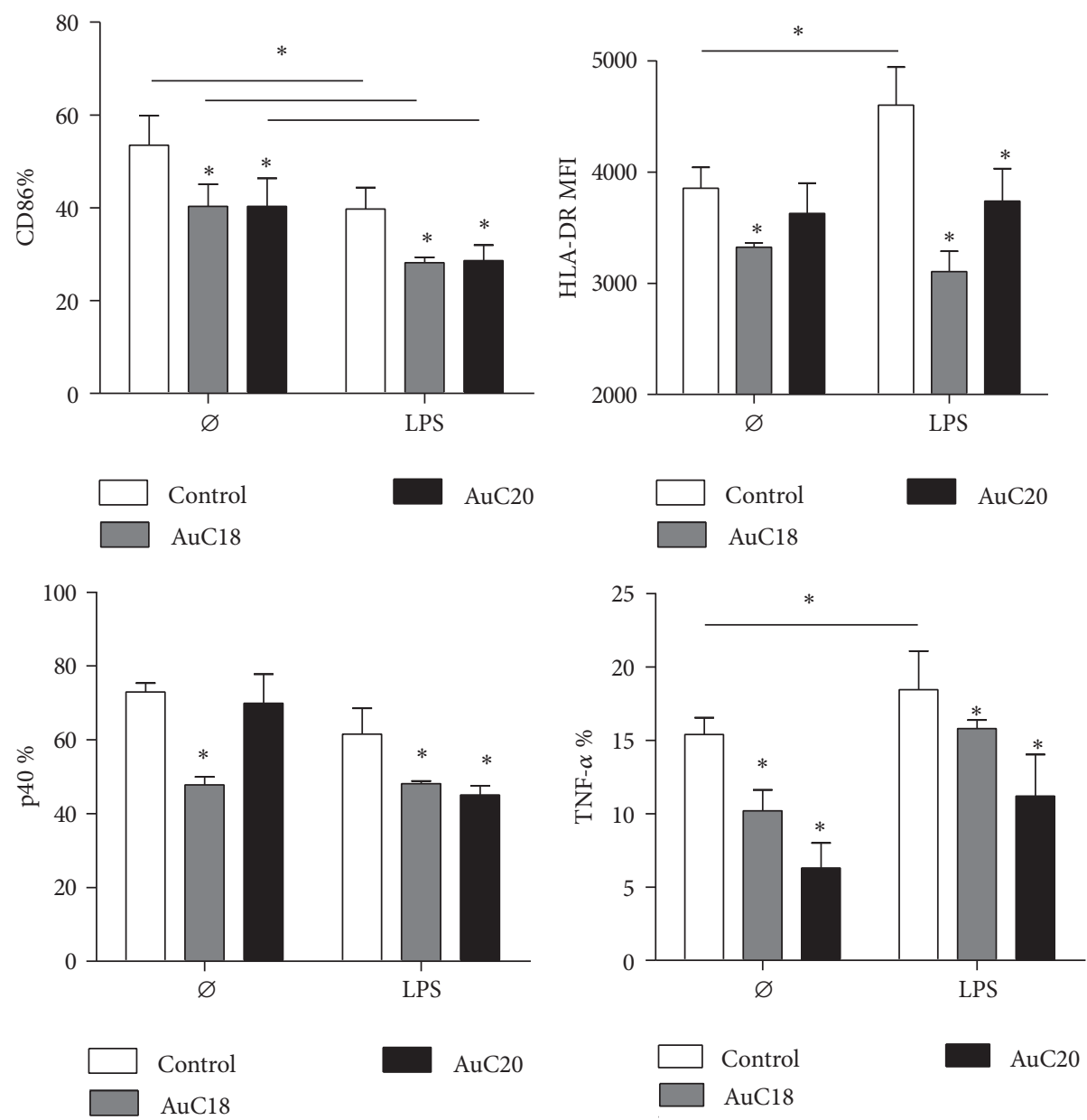

(b)

FIGURE 8: Effect of AuNPs on the expression of CD86, HLA-DR, p40, and TNF- $\alpha$ by human monocytes. (a) Representative histograms of the stained monocytes cultivated in the presence or absence of LPS and AuC18 and AuC20 NP, as indicated. (b) Summarized results are shown as mean $\% \pm \mathrm{SD}(n=3)$ (two-way ANOVA). ${ }^{*} p<0.05$ compared to control.

The number of AuNPs in a given solution could be determined from the ratio between the concentration of AuNPs in a solution and the mass of one AuNP:

$$
N(\text { AuNP })=\frac{C \text { (AuNPs) }}{m(\text { AuNP })}
$$

with $N$ (AuNP) being number of AuNPs, $C$ (AuNP) being concentration of AuNPs as determined by ICP-OES, and $m$ (AuNP) being mass of one AuNP.

The mass of one AuNP can be determined according to the density of gold $\left(\rho=19.32 \mathrm{~g} / \mathrm{cm}^{3}\right)$ and its volume as

$$
m(\mathrm{AuNP})=\rho(\mathrm{Au}) \times \frac{4}{3} \pi r^{3}
$$

with the radius determined by TEM.

$$
\begin{aligned}
N & (\mathrm{AuC18}) \\
& =\frac{14 * 10^{-3}[\mathrm{~g} / \mathrm{L}]}{19.32\left[\mathrm{~g} / \mathrm{cm}^{3}\right] *(4 / 3) \pi *\left(7.35 * 10^{-7}[\mathrm{~cm}]\right)^{3}} \\
& =4.25 * 10^{11} / \mathrm{mL},
\end{aligned}
$$

$$
\begin{aligned}
N & (\text { AuC20 }) \\
& =\frac{4.1 * 10^{-3}[\mathrm{~g} / \mathrm{L}]}{19.32\left[\mathrm{~g} / \mathrm{cm}^{3}\right] *(4 / 3) \pi *\left(1.84 * 10^{-6}[\mathrm{~cm}]\right)^{3}} \\
& =2.71 * 10^{10} / \mathrm{mL} .
\end{aligned}
$$

\section{Abbereviations}

AuNP: Gold nanoparticle

USP: Ultrasonic Spray Pyrolysis

UV-vis: Ultraviolet-visible

TEM: Transmission Electron Microscopy

DLS: Dynamic Light Scattering

APC: Antigen Presenting Cell

DCs: Dendritic cells

ATCC: America Type Culture Collection

RPMI: Roswell Park Memorial Institute

EDTA: Ethylenediaminetetraacetic acid 


$\begin{array}{ll}\text { PBMC: } & \text { Peripheral Blood Mononuclear Cell } \\ \text { LPS: } & \text { Lipopolysaccharide } \\ \text { PBS: } & \text { Phosphate Buffered Saline } \\ \text { SS: } & \text { Side Scatter } \\ \text { SPR: } & \text { Surface Plasmon Resonance } \\ \text { FITC: } & \text { Fluorescein isothiocyanate } \\ \text { PI: } & \text { Propidium Iodide } \\ \text { PE: } & \text { Phycoerythrin } \\ \text { CD: } & \text { Cluster of Differentiation } \\ \text { Ig: } & \text { Immunoglobulin } \\ \text { HLA: } & \text { Human leucocyte antigen } \\ \text { TNF: } & \text { Tumor Necrosis Factor } \\ \text { p40: } & \text { Protein (40) } \\ \text { SD: } & \text { Standard Deviation } \\ \text { EDS: } & \text { Energy Dispersive Spectroscopy (EDS) } \\ \text { ISO: } & \text { International Standard Organization } \\ \text { TLR: } & \text { Toll-like receptor } \\ \text { IL: } & \text { Interleukin } \\ \text { 11-MUDA: } & \text { 1l-Mercaptoundecanoic acid } \\ \text { CT: } & \text { Computed Tomography } \\ \text { LDL: } & \text { Low-density lipids } \\ \text { ox: } & \text { Oxidized } \\ \text { MHC: } & \text { Major Histocompatibility Complex } \\ \text { DNA: } & \text { Deoxyribonucleic acid. } \\ & \end{array}$

\section{Competing Interests}

The authors declare that they have no competing interests.

\section{Acknowledgments}

The study was supported by the Slovenian Research Agency ARRS Slovenia (no. L2-4212 Research Programme P2-0120), the firm Zlatarna Celje d.d., the Ministry of Education, Science and Technological Development of Serbia (no. 175102), and a grant from the Military Medical Academy (MFVMA/08/13-15 and 10/16-18). Thanks go also to Dr. Darja Jenko for performance of the TEM investigations on AuNPs.

\section{References}

[1] P. Pattnaik, "Surface plasmon resonance," Applied Biochemistry and Biotechnology, vol. 126, no. 2, pp. 79-92, 2005.

[2] X. Shi, T. R. Ganser, K. Sun, L. P. Balogh, and J. R. Baker Jr., "Characterization of crystalline dendrimer-stabilized gold nanoparticles," Nanotechnology, vol. 17, no. 4, pp. 1072-1078, 2006.

[3] P. K. Jain, K. S. Lee, I. H. El-Sayed, and M. A. El-Sayed, "Calculated absorption and scattering properties of gold nanoparticles of different size, shape, and composition: applications in biological imaging and biomedicine," The Journal of Physical Chemistry B, vol. 110, no. 14, pp. 7238-7248, 2006.

[4] D. Pissuwan, T. Niidome, and M. B. Cortie, "The forthcoming applications of gold nanoparticles in drug and gene delivery systems," Journal of Controlled Release, vol. 149, no. 1, pp. 65-71, 2011.

[5] M. A. Mahmoud and M. A. El-Sayed, "Different plasmon sensing behavior of silver and gold nanorods," The Journal of Physical Chemistry Letters, vol. 4, no. 9, pp. 1541-1545, 2013.
[6] F. M. Inc, "The Global Market for Gold Nanoparticles, 2010-2025," in Future Markets Inc, 2015, http://www.futuremarketsinc.com/ global-market-gold-nanoparticles-2010-2025/.

[7] Y.-C. Wang and S. Gunasekaran, "Spectroscopic and microscopic investigation of gold nanoparticle nucleation and growth mechanisms using gelatin as a stabilizer," Journal of Nanoparticle Research, vol. 14, no. 10, pp. 1-11, 2012.

[8] R. G. Palgrave and I. P. Parkin, "Aerosol assisted chemical vapor deposition of gold and nanocomposite thin films from hydrogen tetrachloroaurate(III)," Chemistry of Materials, vol.19, no. 19, pp. 4639-4647, 2007.

[9] T. T. T. Kodas and M. J. Hampden-Smith, Aerosol Processing of Materials, Wiley-Vch, Berlin, Germany, 1999.

[10] J. Kimling, M. Maier, B. Okenve, V. Kotaidis, H. Ballot, and A. Plech, "Turkevich method for gold nanoparticle synthesis revisited," The Journal of Physical Chemistry B, vol. 110, no. 32, pp. 15700-15707, 2006.

[11] N. G. Bastús, J. Comenge, and V. Puntes, "Kinetically controlled seeded growth synthesis of citrate-stabilized gold nanoparticles of up to $200 \mathrm{~nm}$ : size focusing versus ostwald ripening," Langmuir, vol. 27, no. 17, pp. 11098-11105, 2011.

[12] G. Schmid and B. Corain, "Nanoparticulated gold: syntheses, structures, electronics, and reactivities," European Journal of Inorganic Chemistry, vol. 2003, no. 17, pp. 3081-3098, 2003.

[13] G. L. Messing, S.-C. Zhang, and G. V. Jayanthi, "Ceramic powder synthesis by spray pyrolysis," Journal of the American Ceramic Society, vol. 76, no. 11, pp. 2707-2726, 1993.

[14] P. Majerič, R. Rudolf, I. Anžel, J. Bogović, S. Stopić, and B. Friedrich, "Synthesis of $\mathrm{NiTi} / \mathrm{Ni}-\mathrm{TiO}_{2}$ composite nanoparticles via ultrasonic spray pyrolysis," Materiali in Tehnologije, vol. 49, no. 1, pp. 75-80, 2015.

[15] A. Baldelli, R. M. Power, R. E. Miles, J. P. Reid, and R. Vehring, "Effect of crystallization kinetics on the properties of spray dried microparticles," Aerosol Science and Technology, vol. 50, no. 7, pp. 693-704, 2016.

[16] J. Dokić, R. Rudolf, S. Tomíc et al., "Immunomodulatory properties of nanoparticles obtained by ultrasonic spray pirolysis from gold scrap," Journal of Biomedical Nanotechnology, vol. 8, no. 3, pp. 528-538, 2012.

[17] R. Rudolf, B. Friedrich, S. Stopić, I. Anžel, S. Tomić, and M. Čolić, "Cytotoxicity of gold nanoparticles prepared by ultrasonic spray pyrolysis," Journal of Biomaterials Applications, vol. 26, no. 5, pp. 595-612, 2012.

[18] S. Stopic, R. Rudolf, J. Bogovic et al., "Synthesis of Au nanoparticles prepared by ultrasonic spray pyrolysis and hydrogen reduction," Materiali in Tehnologije, vol. 47, no. 5, pp. 577-583, 2013.

[19] S. Stopić, Synthesis of Metallic Nanosized Particles by Ultrasonic Spray Pyrolysis, Shaker, Herzogenrath, Germany, 2015.

[20] P. Majerič, B. Friedrich, and R. Rudolf, "Au-nanoparticle synthesis via ultrasonic spray pyrolysis with a separate evaporation zone," Materiali in Tehnologije, vol. 49, no. 5, pp. 791-796, 2015.

[21] P. Majerič, D. Jenko, B. Budič et al., "Formation of nontoxic au nanoparticles with bimodal size distribution by a modular redesign of ultrasonic spray pyrolysis," Nanoscience and Nanotechnology Letters, vol. 7, no. 11, pp. 920-929, 2015.

[22] Y. Qu and X. Lü, "Aqueous synthesis of gold nanoparticles and their cytotoxicity in human dermal fibroblasts-fetalThis work was presented at the 2008 International Materials Research Conference Symposium: Biomaterials for Medical Applications, Chongqing, China, 9-12 June 2008," Biomedical Materials, vol. 4, no. 2, Article ID 025007, 2009. 
[23] N. Khlebtsov and L. Dykman, "Biodistribution and toxicity of engineered gold nanoparticles: a review of in vitro and in vivo studies," Chemical Society Reviews, vol. 40, no. 3, pp. 1647-1671, 2011.

[24] N. Pernodet, X. Fang, Y. Sun et al., "Adverse effects of citrate/gold nanoparticles on human dermal fibroblasts," Small, vol. 2, no. 6, pp. 766-773, 2006.

[25] E. Jan, S. J. Byrne, M. Cuddihy et al., "High-content screening as a universal tool for fingerprinting of cytotoxicity of nanoparticles," ACS Nano, vol. 2, no. 5, pp. 928-938, 2008.

[26] S. Tomić, J. Đokić, S. Vasilijić et al., "Size-dependent effects of gold nanoparticles uptake on maturation and antitumor functions of human dendritic cells in vitro," PLoS ONE, vol. 9, no. 5, Article ID e96584, 2014.

[27] S. Gordon and P. R. Taylor, "Monocyte and macrophage heterogeneity," Nature Reviews Immunology, vol. 5, no. 12, pp. 953-964, 2005.

[28] M. A. Dobrovolskaia and S. E. McNeil, "Immunological properties of engineered nanomaterials," Nature Nanotechnology, vol. 2, no. 8, pp. 469-478, 2007.

[29] J. Bogovic, S. Stopic, B. Friedrich, and J. Schroeder, "Ultrasonic spray pyrolysis," in Proceedings of the EMC, 2011.

[30] P. Pallavicini, A. Donà, A. Casu et al., "Triton X-100 for threeplasmon gold nanostars with two photothermally active NIR (near IR) and SWIR (short-wavelength IR) channels," Chemical Communications, vol. 49, no. 57, pp. 6265-6267, 2013.

[31] T. Zheng, S. Bott, and Q. Huo, “Techniques for accurate sizing of gold nanoparticles using dynamic light scattering with particular application to chemical and biological sensing based on aggregate formation," ACS Applied Materials \& Interfaces, vol. 8, no. 33, pp. 21585-21594, 2016.

[32] Â. Barreto, L. G. Luis, A. V. Girão, T. Trindade, A. M. V. M. Soares, and M. Oliveira, "Behavior of colloidal gold nanoparticles in different ionic strength media," Journal of Nanoparticle Research, vol. 17, no. 12, article no. 493, pp. 1-13, 2015.

[33] X. Huang and M. A. El-Sayed, "Gold nanoparticles: optical properties and implementations in cancer diagnosis and photothermal therapy," Journal of Advanced Research, vol. 1, no. 1, pp. 13-28, 2010.

[34] M. Zhou, B. Wang, Z. Rozynek et al., "Minute synthesis of extremely stable gold nanoparticles," Nanotechnology, vol. 20, no. 50, 2009.

[35] R. Pamies, J. G. H. Cifre, V. F. Espín, M. Collado-González, F. G. D. Baños, and J. G. De La Torre, "Aggregation behaviour of gold nanoparticles in saline aqueous media," Journal of Nanoparticle Research, vol. 16, no. 4, article no. 2376, 2014.

[36] V. A. Dhumale, R. K. Gangwar, S. S. Datar, and R. B. Sharma, "Reversible aggregation control of polyvinylpyrrolidone capped gold nanoparticles as a function of $\mathrm{pH}$," Materials Express, vol. 2, no. 4, pp. 311-318, 2012.

[37] Y. Qu and X. Lü, "Aqueous synthesis of gold nanoparticles and their cytotoxicity in human dermal fibroblasts-fetal," Biomedical Materials, vol. 4, no. 2, Article ID 025007, 2009.

[38] B. D. Chithrani, A. A. Ghazani, and W. C. W. Chan, "Determining the size and shape dependence of gold nanoparticle uptake into mammalian cells," Nano Letters, vol. 6, no. 4, pp. 662-668, 2006.

[39] A. Albanese and W. C. W. Chan, "Effect of gold nanoparticle aggregation on cell uptake and toxicity," ACS Nano, vol. 5, no. 7, pp. 5478-5489, 2011.
[40] R. J. Fahy, A. I. Doseff, and M. D. Wewers, "Spontaneous human monocyte apoptosis utilizes a caspase-3-dependent pathway that is blocked by endotoxin and is independent of caspase-1," Journal of Immunology, vol. 163, no. 4, pp. 1755-1762, 1999.

[41] P. Chhour, P. C. Naha, S. M. O’Neill et al., "Labeling monocytes with gold nanoparticles to track their recruitment in atherosclerosis with computed tomography," Biomaterials, vol. 87, pp. 93103, 2016.

[42] M. A. Vetten, N. Tlotleng, D. Tanner Rascher et al., "Label-free in vitro toxicity and uptake assessment of citrate stabilised gold nanoparticles in three cell lines," Particle and Fibre Toxicology, vol. 10, no. 1, article 50, 2013.

[43] F. Ginhoux and S. Jung, "Monocytes and macrophages: developmental pathways and tissue homeostasis," Nature Reviews Immunology, vol. 14, no. 6, pp. 392-404, 2014.

[44] R. D. Abeles, M. J. McPhail, D. Sowter et al., "CD14, CD16 and HLA-DR reliably identifies human monocytes and their subsets in the context of pathologically reduced HLA-DR expression by CD14hi/CD16neg monocytes: expansion of CD14hi/CD16pos and contraction of CD14lo/CD16pos monocytes in acute liver failure," Cytometry A, vol. 81, no. 10, pp. 823-834, 2012.

[45] D. Strauss-Ayali, S. M. Conrad, and D. M. Mosser, "Monocyte subpopulations and their differentiation patterns during infection," Journal of Leukocyte Biology, vol. 82, no. 2, pp. 244-252, 2007.

[46] N. Thieblemont, L. Weiss, H. M. Sadeghi, C. Estcourt, and N. Haeffner-Cavaillon, "CD14loWCD16high: a cytokineproducing monocyte subset which expands during human immunodeficiency virus infection," European Journal of Immunology, vol. 25, no. 12, pp. 3418-3424, 1995.

[47] G. Fingerle, A. Pforte, B. Passlick, M. Blumenstein, M. Ströbel, and H. W. L. Ziegler-Heitbrock, "The novel subset of CD14+/CD16+ blood monocytes is expanded in sepsis patients," Blood, vol. 82, no. 10, pp. 3170-3176, 1993.

[48] K.-U. Belge, F. Dayyani, A. Horelt et al., "The proinflammatory CD14+CD16+DR++ monocytes are a major source of TNF," Journal of Immunology, vol. 168, no. 7, pp. 3536-3542, 2002.

[49] F. Geissmann, C. Auffray, R. Palframan et al., "Blood monocytes: distinct subsets, how they relate to dendritic cells, and their possible roles in the regulation of T-cell responses," Immunology and Cell Biology, vol. 86, no. 5, pp. 398-408, 2008.

[50] G. J. Randolph, G. Sanchez-Schmitz, R. M. Liebman, and K. Schäkel, "The $\mathrm{CD}^{+} 6^{+}\left(\mathrm{Fc} \gamma \mathrm{RIII}^{+}\right)$subset of human monocytes preferentially becomes migratory dendritic cells in a model tissue setting," The Journal of Experimental Medicine, vol. 196, no. 4, pp. 517-527, 2002.

[51] C. Auffray, D. Fogg, M. Garfa et al., "Monitoring of blood vessels and tissues by a population of monocytes with patrolling behavior," Science, vol. 317, no. 5838, pp. 666-670, 2007.

[52] L. M. Carlin, E. G. Stamatiades, C. Auffray et al., "Nr4a1Dependent Ly6C $\mathrm{C}^{\text {low }}$ monocytes monitor endothelial cells and orchestrate their disposal," Cell, vol. 153, no. 2, pp. 362-375, 2013.

[53] C. A. Hunter, "New IL-12-family members: IL-23 and IL-27, cytokines with divergent functions," Nature Reviews Immunology, vol. 5, no. 7, pp. 521-531, 2005.

[54] M. P. Hayes and K. C. Zoon, "Priming of human monocytes for enhanced lipopolysaccharide responses: expression of alpha interferon, interferon regulatory factors, and tumor necrosis factor," Infection and Immunity, vol. 61, no. 8, pp. 3222-3227, 1993. 
[55] K. Wolk, W.-D. Döcke, V. Von Baehr, H.-D. Volk, and R. Sabat, "Impaired antigen presentation by human monocytes during endotoxin tolerance," Blood, vol. 96, no. 1, pp. 218-223, 2000.

[56] H. Ait-Oufella, S. Taleb, Z. Mallat, and A. Tedgui, "Recent advances on the role of cytokines in atherosclerosis," Arteriosclerosis, Thrombosis, and Vascular Biology, vol. 31, no. 5, pp. 969979, 2011.

[57] A. Fortun, A. Khalil, D. Gagné et al., "Monocytes influence the fate of T cells challenged with oxidised low density lipoproteins towards apoptosis or MHC-restricted proliferation," Atherosclerosis, vol. 156, no. 1, pp. 11-21, 2001. 

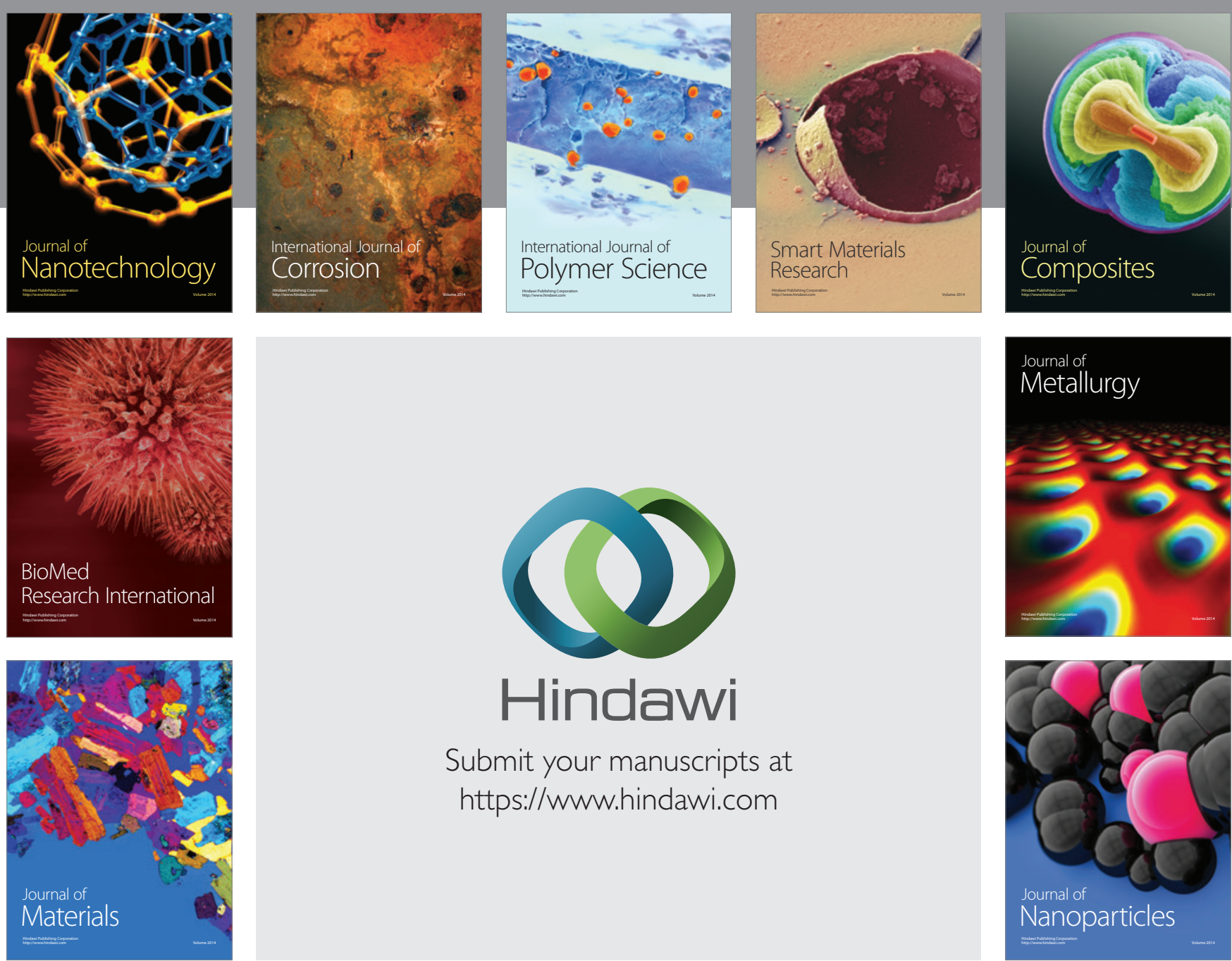

\section{Hindawi}

Submit your manuscripts at

https://www.hindawi.com

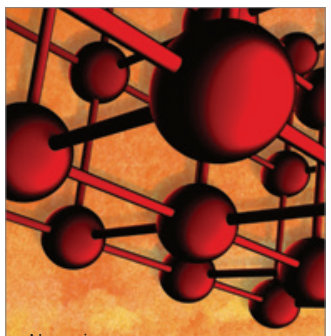

Materials Science and Engineering
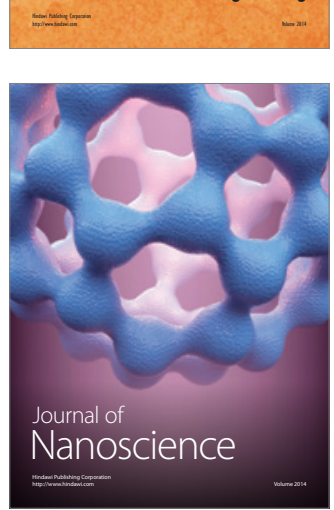
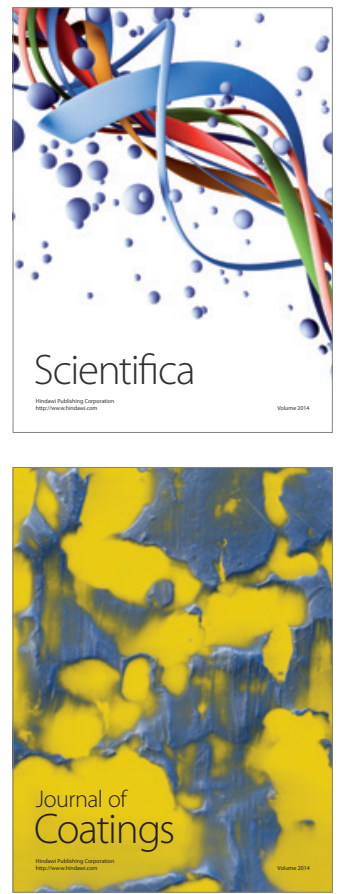
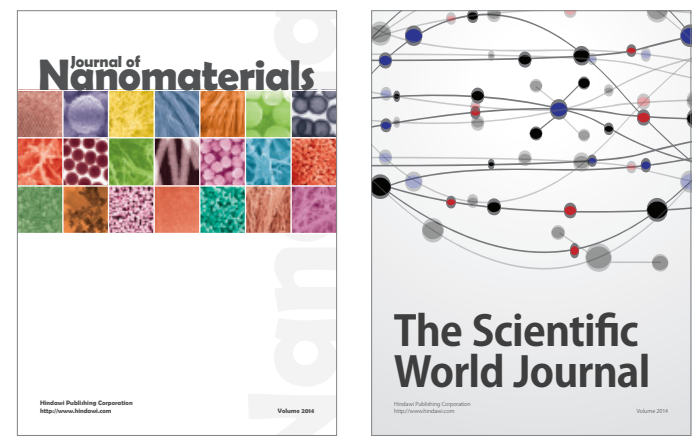

The Scientific World Journal
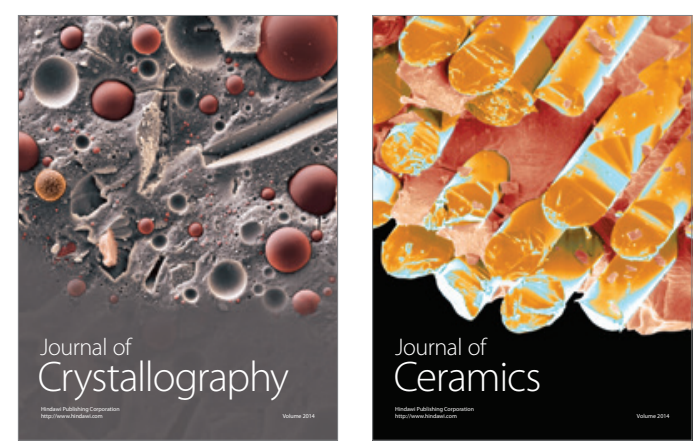
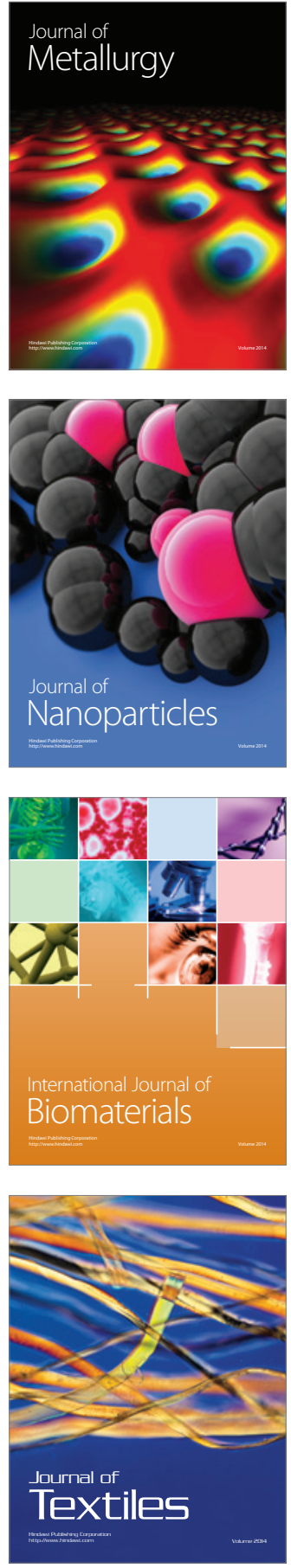